\title{
ANALYTIC FUNCTIONALS WITH UNBOUNDED CARRIERS AND MEAN PERIODIC FUNCTIONS
}

BY

ALEX MERIL

\begin{abstract}
We study certain ideals in some spaces of analytic functionals with unbounded carriers introduced by T. Kawaï, M. Morimoto and J. W. de Roever. Using Banach algebra methods, we show an example of space without spectral synthesis. Using Hörmander's $L^{2}$ estimates, we prove a spectral synthesis theorem for mean periodic functions.
\end{abstract}

Introduction. The Polya-Ehrenpreis-Martineau theorem was proved for analytic functionals with unbounded carriers by J. W. de Roever: Let $\Omega$ be a closed unbounded convex set of $\mathbf{C}^{n}$. He introduced two different spaces of germs of holomorphic functions with growth at infinity. The first, denoted by $\mathscr{H}_{\varepsilon}(\Omega)$, was constructed with the $\varepsilon$-neighborhood of $\Omega$, and the other, $\mathcal{K}_{c}(\Omega)$, using the conic neighborhoods of $\Omega$. Let $a$ be the supporting function of $\Omega$ and $\Gamma$ the "open" convex cone of $\mathbf{C}^{n}, \Gamma=\left\{z \in \mathbf{C}^{n} \mid a(z) \in \mathbf{R}\right\}$. There are two different spaces of analytic functions of exponential type $a$ in $\Gamma$. The first, denoted by $\operatorname{Exp}_{\varepsilon}(\Gamma, a)$, is isomorphic to the dual $\mathcal{H}_{\varepsilon}^{\prime}(\Omega)$ of $\mathcal{H}_{\varepsilon}(\Omega)$, and the other, $\operatorname{Exp}_{c}(\Gamma, a)$, is isomorphic to $\mathcal{H}_{c}^{\prime}(\Omega)$.

In [16] J. W. de Roever points out some applications of these spaces to physics. Hence it is interesting to study convolution equations in these spaces.

In Chapter I, we are interested in convolution equations and mean periodic functions in $\operatorname{Exp}_{\varepsilon}(\Gamma, a)$. By Banach algebra methods, we show an example of a space without spectral synthesis and we characterize some generators of this algebra as follows:

THEOREM. Let $\Omega=\{z \in \mathbf{C}|| \operatorname{Arg} z \mid \leqslant \pi / 4\}$. We have:

$\left(1^{\circ}\right)$ For every $\varphi \in \mathcal{H}_{\varepsilon}(\Omega)$ and every $c>0$ the function $\Psi(z)=e^{-c z^{2}} \varphi(z)$ belongs to $\mathcal{H}_{\varepsilon}(\Omega)$ and the closure of the ideal generated by $\Psi$ is different from $\mathcal{H}_{\varepsilon}(\Omega)$.

$\left(2^{\circ}\right)$ Let $\varphi \in \mathcal{H}_{\varepsilon}(\Omega)$ and $t>0$ such that $\varphi$ is holomorphic and without zero in a $t$-neighborhood of $\Omega$. We also suppose that, for every $c>0, e^{c z^{2}} \varphi \notin \mathcal{H}_{\varepsilon}(\Omega)$. Then the ideal generated by $\varphi$ is dense in $\mathcal{H}_{\varepsilon}(\Omega)$.

Our example is very particular and we do not know if the same is true for other spaces $\operatorname{Exp}_{\varepsilon}(\Gamma, a)$.

In Chapter II, we prove a spectral synthesis theorem in the following manner. As is well known, the study of an invariant subspace is equivalent to the description of the orthogonal $I^{\perp}$ of an ideal $I$ of an algebra $E$ of holomorphic functions on a set $V$.

Received by the editors January 13, 1982.

1980 Mathematics Subject Classification. Primary 46F15, 30M05; Secondary 32C35. 
The description of $I^{\perp}$ is based on the following interpolation problem: Let $\varphi$ be a holomorphic function on the analytic space $\left(X, \vec{c}_{X}\right)$ defined by the sheaf $\Theta / \sigma=\hat{\sigma}_{X}$ (where $\vec{E}$ is the sheaf of germs of holomorphic functions and $\sigma$ is the sheaf of ideals generated by $I$ ). The question is: does the function $\varphi$ belong to $\pi(E)$ (where $\pi$ is the canonical map $\left.\pi: \Gamma(V, \vec{E}) \rightarrow \Gamma\left(X, \vec{E}_{X}\right)\right)$ ? The interest of that question lies in the fact that these spaces are often reflexive spaces. So usually we have $I^{\perp}=(E / I)^{\prime}$ and it is sufficient to study the quotient $E / I$ which we shall try to compare to $\Gamma(V, \mathcal{O}) / \Gamma(V, \widetilde{\mathcal{J}})$.

These interpolation problems are as old as the problems of analysis and spectral synthesis. However, recent works [1, 2] give new methods and interesting results.

The methods of [2] use essentially Hörmander's $L^{2}$ estimates and existence theorems for the $\bar{\partial}$ operator. The methods of [1] are based on integral formulas and projective resolution of the ideal $I$ using Koszul's complexes.

We think that it is possible to solve these problems together (interpolation and description of $I^{\perp}$ ) using $L^{2}$-estimates and existence theorems. That is to say, find a Dolbeault resolution of the spaces $E, I$ and $E / I$.

So the main result in Chapter II is the equivalence between the resolution of these interpolation problems and the existence of exact sequences.

To do so we study the cohomology of a sheaf $K_{0} \Leftrightarrow$ defined on $\mathbf{D}^{2 n}=\mathbf{C}^{n} \amalg S^{2 n-1}$, the spherical compactification of $\mathbf{C}^{n}$ (for $\Omega$ open in $\mathbf{D}^{2 n}$,

$$
\begin{aligned}
& K_{0}(\Omega)=\left\{f \text { holomorphic in } \Omega \cap \mathbf{C}^{n} \text { such that } \forall K \subset \subset \Omega, \forall \varepsilon>0,\right. \\
&\left.\qquad \sup _{z \in K \cap \mathbf{C}^{n}}\left|f(z) \exp \left(-H_{K_{0}}(z)-\varepsilon|z|\right)\right|<+\infty\right\}
\end{aligned}
$$

where $H_{K_{0}}$ is the supporting function of a convex compact set $K_{0}$ of $\mathbf{C}^{n}$ ). We have a soft resolution of $K_{0} \vartheta^{p}$ by sheaves ${ }^{K_{0} \varrho_{2}^{p, q}}$ where ${ }^{K_{0} \varrho_{2}}$ is defined in the same way as $K_{0}\left(\mathcal{C}\right.$, replacing sup norm by $L_{2}$ norm. If $n=1$, we replace the $L_{2}$ spaces by spaces of $C^{\infty}$-functions denoted $\operatorname{Exp}_{c}^{\infty}(\Gamma)$. Now let $I$ be a finitely generated ideal of $\operatorname{Exp}_{c}(\Gamma, 0)$ such that $I=I_{\text {loc }}$ (where $I_{\text {loc }}$ is the local ideal generated by $I$ ). Let $V(I)=$ $\left\{\left(z_{p}, m_{p}\right)_{p \geqslant 0}\right\}$ be the multiplicity variety of the ideal $I$ (see [3] for the definition). We denote by $I_{\infty}$ the ideal

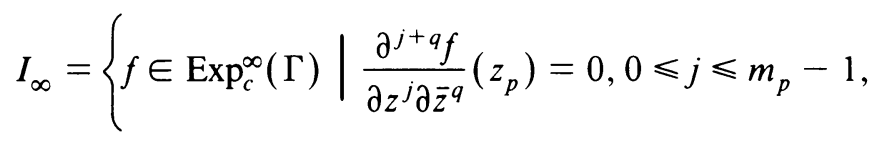

The interpolation theorem is the following:

$$
\text { for every } \left.\left(z_{p}, m_{p}\right) \in V(I) \text { and } q \in \mathbf{N}\right\} \text {. }
$$

THEOREM. Let $I$ be an ideal of $\operatorname{Exp}_{c}(\Gamma, 0)$ and $V(I)=\left\{\left(z_{p}, m_{p}\right)_{p \geqslant 0}\right\}$ be its multiplicity variety. Let $\left(\Gamma_{k}\right)_{k \geqslant 1}$ be an increasing sequence of subcones of $\Gamma$ with union $\Gamma$. Let $\left(a_{p, j}\right)_{p \geqslant 0 ; 0 \leqslant j \leqslant m_{p}-1}$ be a sequence of complex numbers such that for every $k$

$$
\operatorname{Sup}_{\substack{z_{p} \in \Gamma_{k} \\\left|z_{p}\right|>1 / k \\ 0 \leqslant j \leqslant m_{p}-1}}\left|\frac{a_{p, j}}{j !} e^{-\left|z_{p}\right| / k}\right|<+\infty .
$$


Then there is a function $h \in \operatorname{Exp}_{c}(\Gamma, 0)$ such that $\left(\partial^{j} h / \partial z^{j}\right)\left(z_{p}\right)=a_{p, j}(p \in \mathbf{N}$, $0 \leqslant j \leqslant m_{p}-1$ ).

We introduce some technical conditions (in 2.3.6) about slowly decreasing functions. Our main theorem is the following:

THEOREM. With Conditions 2.3.6, the interpolation theorem is equivalent to the exactitude of the sequence

$$
0 \rightarrow \frac{\operatorname{Exp}_{c}(\Gamma, 0)}{I_{\mathrm{loc}}} \stackrel{J}{\rightarrow} \frac{\operatorname{Exp}_{c}^{\infty}(\Gamma)}{I_{\infty}} \stackrel{\bar{\partial}}{\rightarrow} \frac{\operatorname{Exp}_{c}^{\infty}(\Gamma)}{I_{\infty}} \rightarrow 0 .
$$

From this theorem we obtain the following result for mean periodic functions in $\mathcal{H}_{c}(\Omega)$.

THEOREM. Let $\left(\mu_{j}\right)_{1 \leqslant j \leqslant n}$ be $n$ analytic functionals of $\mathcal{H}_{c}^{\prime}(\Omega)$ such that $\left(\mathscr{F}\left(\mu_{j}\right)\right)_{1 \leqslant j \leqslant n}$ verify Conditions 2.3.6 and

$$
I\left(\mathscr{F}\left(\mu_{1}\right), \ldots, \widetilde{\mathscr{F}}\left(\mu_{n}\right)\right)=I_{\text {loc }}\left(\widetilde{F}\left(\mu_{1}\right), \ldots, \widetilde{\mathcal{F}}\left(\mu_{n}\right)\right) .
$$

Then every function $g \in \mathcal{H}_{c}(\Omega)$, such that $\mu_{j} * g=0$ for $1 \leqslant j \leqslant n$, is given by the formula

$$
g(z)=\sum_{p}\left(\sum_{k \leqslant m_{p}} b_{k, p} z^{p}\right) e^{i z \zeta_{p}}
$$

The series is convergent in $\mathcal{H}_{c}(\Omega)$ (where $\left\{\left(\zeta_{p}, m_{p}\right)_{p \geqslant 0}\right\}$ is the multiplicity variety of the ideal $I\left(\widetilde{\mathscr{F}}\left(\mu_{1}\right), \ldots, \widetilde{F}\left(\mu_{n}\right)\right)$.

We would like to thank Professors J. Esterle and R. Gay for valuable discussion and criticism.

CHAPTER I. AN EXAMPLE OF A SPACE WITHOUT SPECTRAL SYNTHESIS

We recall here the definition of the space $\mathcal{H}_{\varepsilon}(\Omega)$ (where $\varepsilon$ is to emphasize that we deal with $\varepsilon$-neighborhoods).

In the rest of the paper, cone will always mean open convex cone of $\mathbf{C}^{n}$ with vertex the origin, and a supporting function on a cone will be a convex and positively homogeneous function of degree one.

Let us recall the following well-known lemma.

1.0.1. LEMMA (SEE [15]). Every closed convex set $\Omega$ of $\mathbf{C}^{n}$ containing no real line determines a cone $\Gamma$ of $\mathbf{C}^{n}$ and a supporting function $a$ (on $\Gamma$ ) such that $\Omega=\left\{\zeta \in \mathbf{C}^{n} \mid\right.$ $-\operatorname{Im}\langle z, \zeta\rangle \leqslant a(z), \forall z \in \Gamma\}$ (with $\left.\langle z, \zeta\rangle=z_{1} \zeta_{1}+\cdots+z_{n} \zeta_{n}\right)$. Conversely any convex cone $\Gamma$ and any supporting function $a$ on $\Gamma$ determines, by the above formula, $a$ closed convex set of $\mathbf{C}^{n}$ containing no real line.

We shall say that $\Omega$ and $(\Gamma, a)$ are in duality and we shall write $\Omega=\Omega(\Gamma, a)$. If $\varepsilon>0$, the $\varepsilon$-neighborhood of $\Omega$ is $\Omega_{\varepsilon}=\Omega(\Gamma, a(z)+\varepsilon|z|)$. 
Let $O$ be a connected open set of $\mathrm{C}^{n}$ and $M$ a continuous function on $O$. We denote by $\mathcal{H}(O)$ the ring of holomorphic functions in $O$ and by $\mathcal{H}(O, M)$ the Banach space

$$
\mathcal{H}(O, M)=\left\{f \in \mathcal{H}(O)\left|\sup _{z \in O}\right| f(z) M(z) \mid<+\infty\right\} .
$$

For an unbounded convex set $\Omega, \mathrm{J}$. W. de Roever generalizes the Polya-EhrenpreisMartineau theorem (see [7]). We recall here the definitions of his spaces.

1.0.2. Definition [16].

(a)

$$
\mathcal{H}_{\varepsilon}(\Omega)=\lim _{k \in \mathbf{N}^{*}} \mathscr{H}\left(\AA_{\Omega_{1 / k}}, \exp \left(\frac{1}{k}|z|\right)\right) .
$$

(b) For $\zeta_{0} \in \Gamma$ the following space is independent from $\zeta_{0}$ :

$$
\operatorname{Exp}_{\varepsilon}(\Gamma, a)=\underset{k \in \mathbf{N}^{*}}{\lim } \mathcal{H}\left(\frac{1}{k} \zeta_{0}+\Gamma, \exp \left(-a(\zeta)-\frac{1}{k}|\zeta|\right)\right) .
$$

Let us remark that the space $\mathscr{H}_{\varepsilon}(\Omega)$ is a D.F.N. space, the $\operatorname{space}_{\operatorname{Exp}}(\Gamma, a)$ is a F.N. space. If $\Omega=\Omega(\Gamma, a)$, then for every $\zeta \in \Gamma$ the function $z \rightarrow e^{i z \zeta}$ belongs to $\mathcal{H}_{\varepsilon}(\Omega)$, so for $\mu \in \mathcal{H}_{\varepsilon}^{\prime}(\Omega)$ we can define $\mathscr{F}(\mu)(\zeta)=\left\langle\mu_{z}, e^{i z \zeta}\right\rangle$, and it is obvious that $\mathscr{F}(\mu) \in \operatorname{Exp}_{\varepsilon}(\Gamma)$. The function $\mathscr{F}(\mu)$ is called the Fourier-Borel transform of the analytic functional $\mu$. The Polya-Ehrenpreis-Martineau type theorem is the following:

1.0.3. THEOREM. The Fourier-Borel transformation $(\mu \rightarrow \mathscr{F}(\mu))$ is a topological isomorphism from $\mathcal{H}_{\varepsilon}^{\prime}(\Omega)$ onto $\operatorname{Exp}_{\varepsilon}(\Gamma, a)$ when $\Omega$ and $(\Gamma, a)$ are in duality.

From now on we shall take $n=1$ and $\zeta_{0}$ will be a fixed point of $\Gamma$. It is obvious that

$$
\mathscr{H}_{\varepsilon}(\Omega)=\underset{\substack{t \rightarrow 0 \\ \varepsilon^{\prime} \rightarrow 0}}{\lim } \mathfrak{H}\left(\Omega_{t}, e^{-i \epsilon^{\prime} \zeta_{1} z}\right)
$$

and

$$
\operatorname{Exp}_{\varepsilon}(\Gamma, a)=\underset{k \in \mathbf{N}^{*}}{\lim } \mathcal{H}\left(\frac{1}{k} \zeta_{0}+\Gamma, \exp \left(-a\left(\zeta-\frac{1}{k} \zeta_{0}\right)-\frac{1}{k}|\zeta|\right)\right)
$$

1.1. The algebra $\mathcal{H}_{\varepsilon}(\Omega)$ and mean periodic function. Let us remark that if $\Omega$ is a closed (unbounded) convex set, $\mathcal{H}_{\varepsilon}(\Omega)$ is a commutative algebra without unit. Since the Fourier-Borel transformation is an isomorphism between $\mathcal{H}_{\varepsilon}^{\prime}(\Omega)$ and $\operatorname{Exp}_{\varepsilon}(\Gamma, a)$, in $\operatorname{Exp}_{\varepsilon}^{\prime}(\Gamma, a)$ there is, as usual, a convolution product such that the transpose ${ }^{t} \mathscr{f}$ of the Fourier-Borel transformation is an algebra isomorphism. We can define the convolution on $\operatorname{Exp}_{\varepsilon}^{\prime}(\Gamma, a)$ in the following way. If $\zeta_{1} \in \Gamma$ and $f \in \operatorname{Exp}_{\varepsilon}(\Gamma, a)$ are given, the function $e_{\zeta_{1}}(f): \zeta \rightarrow f\left(\zeta+\zeta_{1}\right)$ belongs to $\operatorname{Exp}_{\varepsilon}(\Gamma, a)$. So for $R \in$ $\operatorname{Exp}_{\varepsilon}^{\prime}(\Gamma, a)$ we can define the function $R * f$ by $R * f(\zeta)=\left\langle R, \bigodot_{\zeta}(f)\right\rangle$, and it is obvious that $R * f \in \operatorname{Exp}_{\varepsilon}(\Gamma, a)$, and if $R$ and $S$ belong to $\operatorname{Exp}_{\varepsilon}^{\prime}(\Gamma, a)$ then 
$\langle S, R * f\rangle=\langle R, S * f\rangle$. Now for $R$ and $S$ belonging to $\operatorname{Exp}_{\varepsilon}^{\prime}(\Gamma, a)$ we define $R * S \in \operatorname{Exp}_{\varepsilon}^{\prime}(\Gamma, a)$ by $\langle R * S, f\rangle=\langle R, S * f\rangle=\langle S, R * f\rangle$. It is obvious that ${ }^{t} \cdot \bar{r}(R * S)={ }^{t} \widetilde{y}(R)^{t} \cdot \widetilde{y}(S)$ and $R * S=S * R$.

A closed subspace $M$ of $\operatorname{Exp}_{\varepsilon}(\Gamma, a)$ is called invariant (by the translations $己_{\zeta}$ ) if $\mathfrak{E}_{\zeta}(M) \subset M$ for every $\zeta \in \Gamma$. Its orthogonal $M^{\perp}$ is a closed ideal of $\operatorname{Exp}_{\varepsilon}^{\prime}(\Gamma, a)$. Conversely, the orthogonal of a closed ideal of $\operatorname{Exp}_{\varepsilon}^{\prime}(\Gamma, a)$ is an invariant subspace of $\operatorname{Exp}_{f}(\Gamma, a)$. In the same way, the Fourier-Borel transformation gives a bijection between the set of closed ideals of $\mathcal{H}_{\varepsilon}(\Omega)$ and the set of invariant subspaces of $\operatorname{Exp}_{\varepsilon}(\Gamma, a)$. So we only need to study closed ideals of $\mathfrak{K}_{\varepsilon}(\Omega)$. The following problems are classical.

Spectral analysis. Find the exponential monomials (i.e. of the form $z^{p} e^{z \alpha}$ ) belonging to a given invariant subspace $M$.

Spectral synthesis. Study the density in $M$ of the subspace generated by the exponential monomials.

The spectral analysis problem is easy to solve. Following Ehrenpreis [3] we shall set

$$
\operatorname{Spec}(M)=\left\{(z, m) \in \Omega \times \mathbf{N} \mid \zeta^{m} e^{i z \zeta} \in M \text { and } \zeta^{m+1} e^{i z \zeta} \notin M\right\} .
$$

This set is also the multiplicity variety of the ideal $I=t \mathscr{F}\left(M^{\perp}\right)$ (see [3]). The exponential monomials belonging to $M$ are $\zeta^{j} e^{i z \zeta}$ with $0 \leqslant j \leqslant m$ and $(z, m) \in$ $\operatorname{Spec}(M)$.

Let us recall that a function $f \in \operatorname{Exp}_{\varepsilon}(\Gamma, a)$ is called mean periodic if there exists $R \in \operatorname{Exp}_{\varepsilon}^{\prime}(\Gamma, a) \backslash\{0\}$ such that $R * f=0$ (this means that the invariant subspace generated by $\left(\bigodot_{\zeta}(f)\right)_{\zeta \in \Gamma}$ is distinct from $\left.\operatorname{Exp}_{\varepsilon}(\Gamma, a)\right)$.

1.2. Ideals of $\mathcal{H}_{\varepsilon}(\Omega)$. We give here some example of ideals of $\mathcal{H}_{\varepsilon}(\Omega)$. This shall prove that we cannot use the classical proof for the spectral synthesis problem.

1.2.1. RemarK. A principal ideal $\left(f_{0}\right)=f_{0} \mathcal{H}_{\varepsilon}(\Omega)\left(\right.$ with $\left.f_{0} \neq 0\right)$ is never closed.

Proof. There is a problem because $\mathcal{H}_{\varepsilon}(\Omega)$ has no unit and if $f_{0} \neq 0, f_{0} \notin f_{0} \mathcal{H}_{\varepsilon}(\Omega)$. It is easy to see that the ideal generated by $e^{i z \zeta_{1}}\left(\zeta_{1} \in \Gamma\right.$ fixed) is dense in $\mathcal{H}_{\varepsilon}(\Omega)$. In the general case there is $\varepsilon^{\prime}>0$ such that $z \rightarrow f_{0}(z) e^{-i \varepsilon^{\prime} \zeta_{0} z}$ belongs to $\mathcal{H}_{\varepsilon}(\Omega)$, and there is a filter $\left(g_{\alpha}\right)_{\alpha \in A} \subset \mathcal{H}_{\varepsilon}(\Omega)$ such that

$$
e^{i \varepsilon^{\prime} \zeta_{0} z}=\lim _{\alpha} g_{\alpha} e^{i \varepsilon^{\prime} \zeta_{0} z},
$$

so $f_{0}=\lim _{\alpha} f_{0}(z) g_{\alpha}(z)$, that is $f_{0} \in \overline{\left(f_{0}\right)}$.

1.2.2. ExAmple. There is an unbounded convex set $\Omega$ and a function $f \in \mathcal{H}_{\varepsilon}(\Omega), f$ without zeroes in $\Omega$, and for every $\delta>0$ there is $z_{\delta} \in \Omega_{\delta}$ such that $f\left(z_{\delta}\right)=0$. However $\overline{(f)}=\mathfrak{K}_{\varepsilon}(\Omega)$.

Proof. Let us recall the following classical results (see [5]). Let $\left(\beta_{n}\right)_{n \geqslant 1}$ be a sequence of complex numbers such that $\beta_{n} \neq 1$ and $\operatorname{Re} \beta_{n}>0$ for every $n$, and such that $\Sigma_{n \geqslant 0} \operatorname{Re} \beta_{n} /\left(1+\left|\beta_{n}\right|^{2}\right)<+\infty$. The infinite product $F$ defined by

$$
F(w)=\prod_{n \geqslant 1} \frac{\left|1-\beta_{n}^{2}\right|}{1-\beta_{n}^{2}} \frac{w-\beta_{n}}{w+\bar{\beta}_{n}}
$$


is a holomorphic function in $P=\{w \in \mathbf{C} \mid \operatorname{Re} w>0\}$ (called Blaschke product) and $F$ is such that $\operatorname{Sup}_{w \in P}|F(w)| \leqslant 1$.

Let $\Omega=\{z \in \mathbf{C} \mid \operatorname{Re} z \geqslant 1$ and $\operatorname{Im} z \geqslant 1\}$, with $\beta_{n}=1-(1 / n)+i n$. A straightforward calculation gives us that the function $f_{0}(z)=F(z) e^{\varepsilon^{\prime}(i-1) z}\left(\varepsilon^{\prime}>0\right)$ has the properties required by 1.2 .2 .

1.3. Example of a space without spectral synthesis. Here we show an invariant subspace $M \neq\{0\}$ of $\operatorname{Exp}_{\varepsilon}(\Gamma, a)$ without any nonzero exponential monomial, so this space will be without spectral synthesis. This invariant subspace will be of the form $\mathcal{F}\left(I^{\perp}\right)$, where $I$ is the closure in $\mathcal{H}_{\varepsilon}(\Omega)$ of an ideal $(\Psi)$ generated by a function $\Psi$ without zeroes in $\Omega$. Our example is very particular and we do not know what happens in the general case. It seems that the result depends on the geometry of $\Omega$. In this section we shall take

$$
\Omega=\{z \in \mathbf{C}|| \operatorname{Arg} z \mid \leqslant \pi / 4\}
$$

so $\Gamma=\{\zeta \in \mathbf{C} \mid \pi / 4<\operatorname{Arg} \zeta<3 \pi / 4\}$ and $a=0$. We take $\zeta_{0}=i$.

1.3.1. THEOREM. For every $\varphi \neq 0$ and $c>0$, the function $\Psi(z)=e^{-c z^{2}} \varphi(z)$ belongs to $\mathcal{H}_{\varepsilon}(\Omega)$. The closure $I$ of the ideal $(\Psi)$ generated by $\Psi$ in $\mathcal{H}_{\varepsilon}(\Omega)$ is different from $\mathcal{H}_{\varepsilon}(\Omega)$.

Proof. It is obvious that $\Psi \in \mathcal{K}_{\varepsilon}(\Omega)$. If $f \in \mathcal{K}_{t, \varepsilon^{\prime}}(\Omega)=\mathcal{H}_{(}\left(\Omega_{t}, e^{-i \varepsilon^{\prime} \zeta_{0} z}\right)$, we shall denote by $\|f\|_{t, \varepsilon^{\prime}}$ the norm on $\mathcal{H}_{t, \varepsilon^{\prime}}(\Omega)$,

$$
\|f\|_{t, \varepsilon^{\prime}}=\sup _{z \in \Omega_{t}}\left|f(z) e^{-i \varepsilon^{\prime} \zeta_{1} z}\right|=\sup _{z \in \Omega_{t}}\left|f(z) e^{\varepsilon^{\prime} x}\right| \quad(z=x+i y) .
$$

We introduce the sup norm on $\mathcal{H}_{\varepsilon}(\Omega)$ by $P(f)=\sup _{z \in \Omega}|f(z)|$. If $f \in \mathcal{H}_{t, \varepsilon^{\prime}}(\Omega)$ we have $P(f) \leqslant\|f\|_{t, \varepsilon^{\prime}}$ so $P$ is a continuous norm on $\mathcal{H}_{\varepsilon}(\Omega)$.

Let $(H(\Omega), P)$ be the Banach space of holomorphic functions in $\Omega$ continuous on $\Omega$ and vanishing at infinity. It is obvious that $\mathcal{H}_{\varepsilon}(\Omega) \subset H(\Omega)$. We denote by $I_{1}$ the closure of $(\Psi)=\Psi \mathcal{H}_{\varepsilon}(\Omega)$ in $H(\Omega)$. As $I \subset I_{1} \cap \mathcal{H}_{\varepsilon}(\Omega)$, it suffices to prove that $\varphi \notin I_{1}$.

We suppose the opposite. The map $L_{1}$ from $H(\Omega)$ into $H(\Omega), L_{1}(g)=e^{-c z^{2}} g$, is an isometry by the maximum principle, so there is a sequence $\left(f_{n}\right)_{n \in \mathrm{N}}$ of elements of $\mathcal{H}_{\varepsilon}(\Omega)$ such that $\varphi=\lim _{n \rightarrow+\infty} e^{-c z^{2}} \varphi f_{n}$ (in $(H(\Omega), P)$ ). Then there is $\varphi_{1} \in H(\Omega)$ such that $\varphi_{1}=\lim _{n \rightarrow+\infty} \varphi f_{n}$ and $\varphi=e^{-c z^{2}} \varphi_{1}$. By an easy induction using the isometry $L_{m}: g \rightarrow e^{-m c z^{2}} g$ in $H(\Omega)$, we can prove the existence of a sequence $\left(\varphi_{m}\right)_{m \geqslant 1}$ of elements of $H(\Omega)$ such that $e^{-m c z^{2}} \varphi_{m}=\varphi$ for every $m \geqslant 1$ and $P\left(\varphi_{m}\right)=$ $P(\varphi)$. Then we shall have $\varphi=0$, a contradiction.

1.3.2. COROllary. The space $\operatorname{Exp}_{\varepsilon}(\Gamma, a)$ is without spectral synthesis because there is an invariant subspace $M$ containing no nonzero exponential monomial.

Proof. Take $\varphi \in \mathcal{H}_{\varepsilon}(\Omega)$ without zero in $\Omega$ (for example $\varphi(z)=e^{-z}$ ), and for any $c>0$, we note $I=e^{-c z^{2}} \varphi \mathcal{H}_{\varepsilon}(\Omega)$. Then $M=\mathscr{F}\left(I^{\perp}\right)$ has the desired property.

The following theorem characterizes some nontrivial generators of $\mathcal{H}_{\varepsilon}(\Omega)$. 
1.3.3. Theorem. Let $\varphi \in \mathcal{H}_{\varepsilon}(\Omega)$ such that there is $t>0, \varphi \in \mathcal{H}_{(}\left(\Omega_{t}\right), \varphi$ is without zeroes in $\AA_{t}$, and such that, for every $c>0, e^{c z^{2}} \varphi \notin \mathcal{H}_{\varepsilon}(\Omega)$. Then the closure of the ideal $I=\varphi \mathcal{H}_{\varepsilon}(\Omega)$ is equal to $\mathcal{H}_{\varepsilon}(\Omega)$.

Before proving this theorem let us remark that this theorem has been proved in the first part of the proof of 1.2.1 when $\varphi(z)=e^{i z \zeta}(\zeta \in \Gamma)$. So we must point out that there are functions different from an exponential which verify the conditions of 1.3.3 (for example $\varphi(z)=e^{-z} /(z+1)$ ).

Proof of TheOrem 1.3.3. For two strictly positive numbers $\varepsilon^{\prime}$ and $t$, we set

$$
\mathcal{H}_{0, t, \varepsilon^{\prime}}(\Omega)=\left\{f \in \mathcal{K}_{t, \varepsilon^{\prime}}(\Omega) \mid f \text { vanishes at infinity, } f \text { is continuous on } \Omega_{t}\right\} \text {. }
$$

This is a closed subspace of $\mathcal{H}_{t, \varepsilon^{\prime}}(\Omega)$ and it is obvious that

$$
\mathcal{H}_{\varepsilon}(\Omega)=\lim _{t, \varepsilon^{\prime} \rightarrow 0} \mathcal{H}_{0, t, \varepsilon^{\prime}}(\Omega) \text {. }
$$

For a function $f$ on $\Omega_{t}$ we consider the function $g(w)=(f(\sqrt{w}-t \sqrt{2}))$ on $P=\{w \in$ C $\mid \operatorname{Re} w>0\}$ (with $\sqrt{w}=\exp \left(\frac{1}{2} \log w\right)$ we take the principal determination of the logarithm). It is obvious that when $f$ is continuous on $\Omega_{t}$, then $g$ is continuous on $\bar{P}=\{w \mid \operatorname{Re} w \geqslant 0\}$. With this transformation, $\mathcal{H}_{0, t, \varepsilon^{\prime}}(\Omega)$ is isomorphic to the set of functions holomorphic in $\operatorname{Re} w>0$, continuous in $\operatorname{Re} w \geqslant 0$, vanishing at infinity, and such that there is a constant $c_{t, \varepsilon^{\prime}}(g) \geqslant 0$ such that

$$
|g(w)| \leqslant c_{t, \varepsilon^{\prime}}(g) e^{-\varepsilon^{\prime} \operatorname{Re} \sqrt{w}} \quad(\operatorname{Re} w \geqslant 0) .
$$

In the following, $t$ and $\varepsilon^{\prime}$ are such that $\varphi \in \mathcal{H}_{0, t, \varepsilon^{\prime}}(\Omega)$ and $\varphi$ is without zeroes in $\Omega_{t}$. We denote by $\Phi$ the function $\Phi(w)=\varphi(\sqrt{w}-t \sqrt{2})$, and, for $0<\varepsilon_{1}^{\prime}<\varepsilon^{\prime}$,

$$
g(w)=e^{\varepsilon_{1}^{\prime} \sqrt{w}} \Phi(w) / c_{t, \varepsilon^{\prime}}(\Phi),
$$

so $|g(w)| \leqslant 1$ for every $w$. Using a classical result (see [5]) $g$ can be written $g=\lambda B S F$ where $\lambda \in \mathbf{C}$ and $|\lambda|=1, B$ is a Blaschke product (here $B=1$ because $g$ has no zeroes), $F$ is an outer function and $S$ is an inner function. The outer function $F$ is given by the formula

$$
F(w)=\exp \left(\frac{1}{\pi} \int_{-\infty}^{+\infty} \log |g(i t)| \frac{t w+i}{t+i w} \frac{d t}{1+t^{2}}\right),
$$

and $S(w)=e^{-c w}(c \geqslant 0)$ because $g$ is continuous in $\operatorname{Re} w \geqslant 0$. But $|F(w)| \leqslant 1$ so $c=0$. Suppose on the contrary that $c>0$. Then we deduce obviously that $e^{c z^{2}} \varphi \in$ $\mathcal{H}_{\varepsilon}(\Omega)$, a contradiction. Then

$$
\Phi(w)=\lambda c_{t, \varepsilon^{\prime}}(\Phi) e^{-\varepsilon_{1}^{\prime} \sqrt{w}} F(w)
$$

and a straightforward calculation shows that

$$
\Phi(w)=\lambda \operatorname{Exp}\left(\frac{1}{\pi} \int_{-\infty}^{+\infty} \log |\Phi(i t)| \frac{t w+i}{t+i w} \frac{d t}{1+t^{2}}\right) .
$$

We introduce here proximate units, setting

$$
\Psi_{n}(w)=\frac{1}{\lambda} \operatorname{Exp}\left(-\frac{1}{\pi} \int_{-n}^{+n} \log |\Phi(i t)| \frac{t w+i}{t+i w} \frac{d t}{1+t^{2}}\right) .
$$


These functions are bounded on $\operatorname{Re} w \geqslant 0$. Now we set $\theta_{n}(z)=\Psi_{n}\left((z+t \sqrt{2})^{2}\right)$. It is obvious that for every $h \in \mathcal{H}_{\varepsilon}(\Omega), h \theta_{n} \in \mathcal{H}_{\varepsilon}(\Omega)$ and $h \theta_{n} \varphi \in \mathcal{H}_{\varepsilon}(\Omega)$. A straightforward calculation gives $h=\lim _{n \rightarrow+\infty} h \theta_{n} \varphi$ for any $h \in \mathcal{H}_{\varepsilon}(\Omega)$.

CHAPTER II. INTERPOLATION AND MEAN PERIODIC FUNCTIONS

Let $\Gamma$ be a cone of $\mathbf{C}^{n}$. As usual $S^{2 n-1}$ is the unit sphere of $\mathbf{C}^{n}$ and we note $\operatorname{pr} \Gamma=\Gamma \cap S^{2 n-1}$. If $\Gamma$ and $\Gamma^{\prime}$ are two cones we write $\Gamma \subset \subset \Gamma^{\prime}$ if $\operatorname{pr} \Gamma \subset \subset \Gamma^{\prime}$.

An exhaustion of a cone $\Gamma$ is an increasing family of subcones $\left(\Gamma_{k}\right)_{k \geqslant 1}$ with union $\Gamma$ and such that for every $k, \Gamma_{k} \subset \subset \Gamma_{k+1} \subset \subset \Gamma$.

Let $a$ be a supporting function in $\Gamma, \Omega=\Omega(\Gamma, a)$, and $\left(\Gamma_{k}\right)_{k \geqslant 1}$ an exhaustion of $\Gamma$. We set $\Omega_{c}^{k}=\Omega\left(\Gamma_{k}, a+|z| / k\right)$ (the subscript $c$ stands for conic), and the space $\mathcal{H}_{c}(\Omega)$ is given by the following:

2.0.1. DeFinition.

$$
\mathscr{H}_{c}(\Omega)=\lim _{\vec{k}} \mathcal{H}\left(\Omega_{c}^{k}, \exp \left(\frac{1}{k}|z|\right)\right) .
$$

(It is obvious that the space $\mathcal{H}_{c}(\Omega)$ is independent from the given exhaustion of $\Gamma$.)

Similarly, we define the $\operatorname{space}_{\operatorname{Exp}_{c}}(\Gamma, a)$ by

2.0.2. Definition. We set $\Gamma(k)=\Gamma_{k} \cap\{z|| z \mid>(1 / k)\}$ and define

$$
\operatorname{Exp}_{c}(\Gamma, a)=\underset{\leftarrow}{\lim } \mathcal{H}\left(\Gamma(k), \exp \left(-a(z)-\frac{1}{k}|z|\right)\right) .
$$

This space is independent from the given exhaustion of $\Gamma$. This is a F.N. space, $\mathcal{H}_{c}(\Omega)$ is a D.F.N. space. J. W. de Roever proves a Polya-Martineau-Ehrenpreis type theorem for this space (see Chapter I).

2.0.3. THEOREM [16]. The Fourier-Borel transformation is a topological isomorphism between $\mathcal{H}_{c}^{\prime}(\Omega)$ and $\operatorname{Exp}_{c}(\Gamma, a)$.

Now if $a=0$, the space $\operatorname{Exp}_{c}(\Gamma, a)$ is a ring, so as in Chapter I, we can define the notion of an invariant subspace of $\mathcal{H}_{c}(\Omega(\Gamma, a))$. There are also two fundamental problems (spectral analysis and spectral synthesis).

RemarK. The space $\operatorname{Exp}_{c}(\Gamma, a)$ is different from the $\operatorname{space}_{\operatorname{Exp}_{\varepsilon}}(\Gamma, a)$ of Chapter I.

2.1. Quotient of functions of exponential type. To study mean periodic functions in a space of holomorphic functions, usually theorems analogous to Lindelöff's famous division theorem are needed (if $f$ and $g$ are entire functions of exponential type and if $f / g$ is entire, then $f / g$ is also of exponential type) (see [3 and 18]). We show here that these techniques cannot be used for mean periodic functions in $\mathcal{H}_{c}(\Omega(\Gamma, a))$. The counterexample is the following:

The function

$$
g(z)=\frac{1}{\Gamma_{1}(z)}=z e^{\gamma z} \prod_{n \geqslant 1}\left(1+\frac{z}{n}\right) e^{-(z / n)}
$$

( $\gamma$ is Euler's constant and $\Gamma_{1}$ is Euler's gamma function) is entire of order 1 and not of exponential type in $\mathbf{C}$, but Stirling's formula shows that

$$
|g(z)| \leqslant c_{1}(|z| / e)^{(1 / 2)-\operatorname{Re} z} e^{(\pi / 2)|\operatorname{Im} z|}
$$

so $g \in \operatorname{Exp}_{c}(\Gamma, 0)$ with $\Gamma=\{z \in \mathbf{C}|| \operatorname{Arg} z \mid<\pi / 4\}$. 
If a function $f$ is holomorphic in a cone $\Gamma$, we shall say that $f$ is of exponential type in $\Gamma$, if there is a supporting function $a$ on $\Gamma$ such that $f \in \operatorname{Exp}_{c}(\Gamma, a)$. So $g$ is of exponential type in $\{z \in \mathbf{C}|| \operatorname{Arg} z \mid<\pi / 4\}$. However, 1/g (Euler's gamma function) is not.

However we have the following positive results.

2.1.1. THEOREM (SEE [8]). If $f$ is an entire function of exponential type (on $\mathbf{C}$ ) and if $C$ is a cone such that $f$ is without zeroes in $C$, then $1 / f$ is of exponential type on $C$.

Following Malgrange [12] we can easily prove the following:

2.1.2. Proposition. Let $f$ and $g$ be two entire functions (on $\mathbf{C}$ ) of exponential type. Let $\left(\alpha_{n}\right)_{n \geqslant 1}$ be the sequence of common zeroes of $f$ and $g$ in a convex open sector $C$. If $f / g$ is holomorphic in $C$ and if $\sup _{n}\left|\sum_{p=1}^{n} 1 / \alpha_{p}\right|<+\infty$, then $f / g$ is of exponential type in $C$.

2.2. The sheaf $K_{0} \vartheta$ and its cohomology. We note $\mathbf{D}^{2 n}$, the spherical compactification of $\mathbf{C}^{n}$, defined by

$$
S_{\infty}^{2 n-1}=\left\{U_{\infty} \mid U \in S^{2 n-1}\right\}
$$

and $\mathbf{D}^{2 n}=\mathbf{C}^{n} \amalg S_{\infty}^{2 n-1}$ (disjoint union). The topology of $\mathbf{D}^{2 n}$ is the following:

$\left(1^{\circ}\right)$ A base of neighborhoods of a point $x \in \mathbf{C}^{n}$ is given by the open balls containing $x$.

$\left(2^{\circ}\right)$ A base of neighborhoods of a point $U_{\infty} \in S_{\infty}^{2 n-1}$ is given by the sets $(z+\Gamma) \amalg(\operatorname{pr} \Gamma)_{\infty}$ with $z \in \mathbf{C}^{n}, \Gamma$ an open convex cone of $\mathbf{C}^{n}$ such that $U_{\infty} \in(\operatorname{pr} \Gamma)_{\infty}$ (that is $U \in \operatorname{pr} \Gamma=\Gamma \cap S^{2 n-1}$ ).

From now on, $K_{0}$ will be a convex compact set of $\mathbf{C}^{n}$ such that $0 \in K_{0}$. We note $a=H_{K_{0}}$ the supporting function of $K_{0}$. Let us recall that $H_{K_{0}}$ is defined by $H_{K_{0}}(z)=\sup _{\zeta \in K_{0}}-\operatorname{Im}\langle z, \zeta\rangle$. We take $K_{0}$ such that $a \in \mathcal{C}^{1}\left(\mathbf{C}^{n} \backslash\{0\}\right)$.

2.2.1. Definition. For every open set $\Omega$ of $\mathbf{D}^{2 n}$, we set

$\kappa_{0} \Theta(\Omega)=\left\{\right.$ f holomorphic in $\Omega \cap \mathbf{C}^{n} \mid \forall K \subset \subset \Omega, \forall \varepsilon>0$,

$$
\left.\sup _{z \in K \cap \mathbf{C}^{n}}|f(z) \exp (-a(z)-\varepsilon|z|)|<+\infty\right\} \text {. }
$$

It is obvious that we have a sheaf ${ }^{K_{0}} \theta$ such that $\left.{ }^{K_{0}} \theta\right|_{\mathbf{C}^{n}}=\theta$ (the sheaf of germs of holomorphic functions on $\mathbf{C}^{n}$ ).

Let us recall that

$$
\operatorname{Exp}\left(K_{0}\right)=\left\{f \in \mathcal{H}\left(\mathbf{C}^{n}\right)\left|\forall \varepsilon>0, \sup _{z \in \mathbf{C}^{n}}\right| f(z) \exp (-a(z)-\varepsilon|z|) \mid<+\infty\right\}
$$

is the well-known space of entire functions of "exponential type $H_{K_{0}}$ ". This is a F.S. space as projective limit of the following Banach spaces:

$$
\operatorname{Exp}\left(K_{0}, \varepsilon\right)=\left\{f \in \mathcal{H}\left(\mathbf{C}^{n}\right)\left|\sup _{z \in \mathbf{C}^{n}}\right| f(z) \exp (-a(z)-\varepsilon|z|) \mid<+\infty\right\} .
$$

It is obvious that $\operatorname{Exp}\left(K_{0}\right)=\Gamma\left(\mathbf{D}^{2 n}, K_{0} \vartheta\right)$. Let us also recall that $\operatorname{Exp}\left(K_{0}\right)$ is isomorphic to $\mathcal{H}^{\prime}\left(K_{0}\right)$ by the Fourier-Borel transformation where $\mathcal{H}^{\prime}\left(K_{0}\right)$ is the 
space of analytic functionals carried by $K_{0}$ (this is the Polya-Ehrenpreis-Martineau theorem, see [7]).

If $C$ is an open convex cone of $\mathbf{C}^{n}$, it is obvious that we have

$$
\operatorname{Exp}_{c}(C, a)=\Gamma\left(C \amalg(\operatorname{pr} C)_{x}, K_{0} \Theta\right)=K_{0} \Theta\left(C \amalg(\operatorname{pr} C)_{\infty}\right) .
$$

The following sheaves will be useful to find the cohomology of $K_{0} \theta$.

2.2.2. Definition. For every open set $\Omega$ of $\mathbf{D}^{2 n}$, we set

$$
\begin{aligned}
K_{0} \varrho_{2}(\Omega)=\left\{f \in L_{\mathrm{loc}}^{2}\left(\Omega \cap \mathbf{C}^{n}\right) \mid \forall K \subset \subset \Omega, \forall \varepsilon>0,\right. \\
\left.\qquad \int_{K \cap \mathbf{C}^{n}}|f(z)|^{2} e^{-a(z)-\varepsilon|z|} d \lambda(z)<+\infty\right\} .
\end{aligned}
$$

If $\mathscr{F}$ is a sheaf on $\mathbf{D}^{2 n}$, we shall, as usual, denote by $\mathscr{F}^{p, q}$ the sheaf of differential forms of bidegree $(p, q)$ with coefficients in $\mathscr{F}$.

When it is possible, we shall use the classical $\bar{\partial}$ operator $($ see $[6,7])$ defined by

$$
\bar{\partial} F=\sum_{k=1}^{n} \sum_{\substack{|I|=p \\|J|=q}} \frac{\partial F_{I, J}}{\partial \bar{z}_{k}} d \bar{z}_{k} \wedge d Z_{I} \wedge d \bar{Z}_{J}
$$

if

$$
F=\sum_{\substack{|I|=p \\|J|=q}}^{\prime} F_{I, J} d Z_{I} \wedge d \bar{Z}_{J}
$$

2.2.3. Definition. For every open set $\Omega$ of $\mathbf{D}^{2 n}$ we set

$$
\left.K_{0}\right) \mathcal{H}^{p, q}(\Omega)=\left\{f \in K^{\prime \prime} \varrho_{2}^{p, q}(\Omega) \mid \bar{\partial} f \in K_{10} \varrho_{2}^{p, q+1}(\Omega)\right\}
$$

( $\bar{\partial}$ is defined here in the sense of distribution theory).

2.2.4. Proposition. The presheaves ${ }^{0} \mathcal{H}^{p, q}$ are soft sheaves.

Proof. For every open set $\Omega$ of $\mathbf{D}^{2 n}$, we set

$\mathcal{Q}(\Omega)=\left\{f \in e^{\infty}\left(\Omega \cap \mathbf{C}^{n}\right) \mid \forall K \subset \subset \Omega\right.$,

$$
\left.\operatorname{Sup}\left(\operatorname{Sup}_{\zeta \in K \cap \mathbf{C}^{n}}|f(\zeta)|, \sup _{\zeta \in K \cap \mathbf{C}^{n}} \sum_{j=1}^{n}\left|\frac{\partial f}{\partial \bar{z}_{j}}(\zeta)\right|\right)<+\infty\right\} .
$$

It is obvious that $\mathcal{Q}$ is a soft sheaf of rings with unit and that $K_{0} \mathcal{F}^{p . q}$ is an $\mathcal{Q}$-module, so $K_{0} \mathcal{F C}^{p, q}$ is soft.

To study the cohomology of $K_{0} \vartheta$, we need the following: An open set $O$ of $\mathbf{C}^{n}$ has property $(\mathrm{P})$ if it is connected and if there is a holomorphic function $\varphi$ in $O$ such that for every $\delta>0$ we have $\sup _{z \in O}(-\operatorname{Re} \varphi(z)+\delta|z|)<+\infty$.

The following sets have this property:

(a) for $n=1$ :

$\left(1^{\circ}\right) O=z_{0}+\xi_{0} O_{1}$ with $\left|\xi_{0}\right|=1, z_{0} \in \mathbf{C}$ and

$$
O_{1}=\{z \in \mathbf{C}|| \operatorname{Arg} z \mid<\beta<\pi / 2\}
$$


and

$$
\varphi(\zeta)=\left(\frac{\zeta-z_{0}}{\xi_{0}}\right)^{1+\varepsilon} \quad\left((1+\varepsilon) \beta<\frac{\pi}{2}\right) ;
$$

$\left(2^{\circ}\right) O=\xi_{0} O_{1}$ with $\left|\xi_{0}\right|=1, \sup _{z \in O_{1}}|\operatorname{Im} z|<+\infty$ and $\varphi(z)=\left(z / \xi_{0}\right)^{2}$;

(b) for $n \geqslant 1$ :

$\left(1^{\circ}\right) O$ is bounded and $\varphi \equiv 1$;

$\left(2^{\circ}\right) O=\Gamma_{1} \times \cdots \times \Gamma_{n}$ with $\Gamma_{k}=z_{0}^{k}+\xi_{0}^{k} O_{1}^{k}, z_{0}^{k} \in \mathbf{C}, \xi_{0}^{k} \in \mathbf{C},\left|\xi_{0}^{k}\right|=1$,

$$
O_{1}^{k}=\left\{z_{k} \in \mathbf{C}|| \operatorname{Arg} z_{k} \mid<\beta_{k}<\pi / 2\right\}
$$

and $\varphi=\varphi_{1}+\cdots+\varphi_{n}$, where

$$
\varphi_{k}(z)=\left(\frac{z_{k}-z_{0}^{k}}{\xi_{0}^{k}}\right)^{1+\varepsilon_{k}} \quad\left(\left(1+\varepsilon_{k}\right) \beta_{k}<\frac{\pi}{2}, z=\left(z_{1}, \ldots, z_{n}\right)\right) .
$$

The following theorem is analogous to Lemma 2.1.1 of Kawaï [8]. Related results have been announced by Saburi [17] but, as far as we know, his proof has never appeared.

2.2.5. THEOREM. Let $\Omega$ be an open set of $\mathbf{D}^{2 n} \backslash\{0\}$, $\sigma$ a strictly plurisubharmonic function belonging to $\varrho^{2}\left(\Omega \cap \mathbf{C}^{n}\right)$. We set $L_{j}=\left\{z \in \Omega \cap \mathbf{C}^{n} \mid \sigma(z)<j\right\}$ and $K_{j}$ the closure of $L_{j}$ in $\mathbf{D}^{2 n}$. We choose $\sigma$ such that $K_{j} \cap \mathbf{C}^{n}=L_{j}$. We suppose also that the boundary $\partial L_{j}$ is $\left\{z \in \Omega \cap \mathbf{C}^{n} \mid \sigma(z)=j\right\}$ and is $\varrho^{2}$, that the family of sets $\left(K_{j}\right)_{j \geqslant 1}$ is an exhaustion of $\Omega$, and $\Omega \cap \mathrm{C}^{n}$ has property $(\mathrm{P})$. Then the following sequence is exact:

$$
K_{0} \varrho_{2}^{p .0}(\Omega) \stackrel{T^{0}}{\rightarrow} K_{0} \varrho_{2}^{p, 1}(\Omega) \stackrel{T^{1}}{\rightarrow} \cdots \stackrel{T^{n-1}}{\rightarrow} K_{0} \varrho_{2}^{p, n}(\Omega) \rightarrow 0
$$

(where $T^{q}:{ }^{K_{0}} \varrho_{2}^{p, q}(\Omega) \rightarrow{ }^{K_{0}} \varrho_{2}^{p, q+1}(\Omega)$ is the densely defined operator of domain

$$
D\left(T^{q}\right)=\left\{f \in K_{0} \varrho_{2}^{p, q}(\Omega) \mid \bar{\partial} f \in K_{0} \varrho_{2}^{p, q+1}(\Omega)\right\}
$$

and $T^{q} f=\bar{\partial} f$ in the sense of distribution theory).

Proof. Recall that if $\varphi$ is a weight function on $A \subset \mathbf{C}^{n}$ we note

$$
L_{2}(A, \varphi)=\left\{\left.f \in L_{\mathrm{loc}}^{1}(A)\left|\int_{A}\right| f\right|^{2} e^{-\varphi} d \lambda<+\infty\right\},
$$

this space being a Hilbert space under the norm

$$
\|f\|_{L_{2}(A, \varphi)}^{2}=\int_{A}|f|^{2} e^{-\varphi} d \lambda
$$

( $\lambda$ is the Lebesgue measure on $C^{n}$ ). We denote by $L_{2}^{p, q}(A, \varphi)$ the Hilbert space of $(p, q)$ forms with coefficients in $L_{2}(A, \varphi)$ with norm

$$
\|f\|^{2}=\sum_{I, J}^{\prime}\left\|f_{I, J}\right\|_{L^{2}(A, \varphi)} \text { if } f=\sum_{I, J}^{\prime} f_{I, J} d Z_{I} \wedge d \bar{Z}_{J} .
$$

For every $m \in \mathbf{N}$ and $j \in \mathbf{N} \backslash\{0\}$ we let

$$
X_{j, m}^{p, q}=L_{2}^{p, q}\left(\stackrel{\circ}{K}_{j} \cap \mathbf{C}^{n}, j^{-1}|z|+a(z)+2 m \log \left(1+|z|^{2}\right)\right) .
$$


The space $K_{0} \varrho_{2}^{p, q}(\Omega)$ is equal to the projective $\operatorname{limit} \lim _{j} X_{j, m}^{p, q}$ for every $m \geqslant 0$. It is obvious that $K_{0} \varrho_{2}^{p, q}(\Omega) \subset \lim _{-j} X_{j, m}^{p, q}$. On the other hand, let $K$ be a compact subset of $\Omega, \varepsilon$ a strictly positive number, and $j \in \mathbf{N}$ large enough so that $K \subset \stackrel{\circ}{K}_{j}$ and $1 / j<\varepsilon$. We have, for every $f \in X_{j, m}^{p, 0}$,

$$
\int_{K \cap \mathbf{C}^{n}}|f(z)|^{2} e^{-u(z)-\varepsilon|z|} d \lambda(z) \leqslant \frac{1}{M} \int_{K, \cap \mathbf{C}^{n}} \frac{|f(z)|^{2} e^{-a(z)-|z| / j}}{\left(1+|z|^{2}\right)^{2 m}} d \lambda(z)
$$

with

$$
M=\inf _{x \in \mathbf{R}_{+}} \frac{e^{(\varepsilon-1 / j) x}}{\left(1+x^{2}\right)^{2 m}}
$$

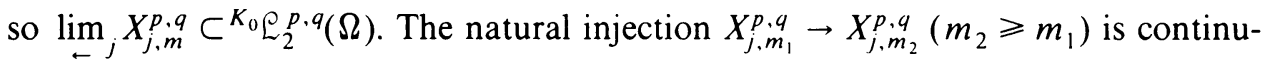
ous, so the identity map $\lim _{\leftarrow} X_{j, m_{1}}^{p, q} \rightarrow \lim _{-j} X_{j, m_{2}}^{p, q}$ is continuous. Thus the different topologies of F.S.* spaces (see [9]) coincide on $K_{0} \varrho_{2}^{p, q}(\Omega)$ by the open mapping theorem.

The dual of $X_{j, m}^{p, q}$ is equal to the space

$$
L_{2}^{p, q}\left(\stackrel{\circ}{K}_{j} \cap \mathbf{C}^{n},-a(z)-j^{-1}|z|-2 m \log \left(1+|z|^{2}\right)\right),
$$

where the duality is given by

$$
\langle f, g\rangle=\sum_{\substack{|I|=p \\|J|=q}}^{\prime} \int_{K, \cap \mathbf{C}^{n}} f_{I, J} g_{I, J} d \lambda
$$

if

$$
f=\sum_{\substack{|I|=p \\|J|=q}}^{\prime} f_{I, J} d Z_{I} \wedge d \bar{Z}_{J} \text { and } g=\sum_{\substack{|I|=p \\|J|=q}}^{\prime} g_{I, J} d Z_{I} \wedge d \bar{Z}_{J}
$$

We denote by $T_{j, m}^{q}$ the densely defined operator $T_{j, m}^{q}(f)=\bar{\partial} f$ with domain

$$
D\left(T_{j, m}^{q}\right)=\left\{f \in X_{j, m}^{p, q} \mid \bar{\partial} f \in X_{j, m-1}^{p, q+1}\right\} \quad(m \geqslant 1) .
$$

For every $q \geqslant 1$ and $m \geqslant 2$ the following sequence is exact (see [6 and 7]):

$$
X_{j, m+1}^{p, q-1} \stackrel{T_{j, m+1}^{q-1}}{\rightarrow} X_{j, m}^{p, q} \stackrel{T_{j, m}^{q}}{\rightarrow} X_{j, m-1}^{p, q+1} .
$$

The operator $T_{j, m}^{q}$ has a closed range because $\operatorname{Im} T_{j, m}^{q}=\operatorname{Ker} T_{j, m-1}^{q}$. This operator has a dual operator (see [10]), denoted ${ }^{t} T_{j, m}^{q}$, and given by

$$
{ }^{t} T_{j, m}^{q}(f)=(-1)^{p-1} \sum_{\substack{|I|=p \\|M|=q}} \sum_{k} \frac{\partial f_{I, k M}}{\partial \bar{z}_{k}} d Z_{I} \wedge d \bar{Z}_{M}
$$

if

$$
f=\sum_{\substack{|I|=p \\|J|=q+1}}^{\prime} f_{I, J} d Z_{I} \wedge d \bar{Z}_{J}
$$


The dual sequence of $(1)_{j}$,

$$
\left(X_{j, m+1}^{p, q-1}\right)^{\prime} \stackrel{{ }^{\prime} T_{j, m+1}^{q-1}}{\rightarrow}\left(X_{j, m}^{p, q}\right)^{\prime} \stackrel{{ }^{\prime} T_{j, m}^{q}}{\leftarrow}\left(X_{j, m-1}^{p, q+1}\right)^{\prime}
$$

is also exact by the Serre-Komatsu lemma [9].

For every $k \geqslant j, s_{j, k, m}^{p, q}$ is the restriction map: $X_{k, m}^{p, q} \stackrel{s_{j, q . m}^{p, q} \rightarrow}{\rightarrow} X_{j, m}^{p, q}$. Its transposed map is "the extension by zero".

A straightforward calculation shows that the system $\left(\left(X_{j, m}^{p, q}\right)^{\prime}, s_{j, k, m}^{p, q}\right)_{k \geqslant j:(k, j) \in \mathbf{N}^{2}}$ is inductive, that we can take the inductive limit of the sequence $(2)_{j}$, and that we also obtain an exact sequence

$$
\lim _{\vec{j}}\left(X_{j, m+1}^{p, q-1}\right)^{\prime}{ }^{\prime} T_{m}^{q-1} \leftarrow \lim _{\vec{j}}\left(X_{j, m}^{p, q}\right)^{\prime}{ }^{\prime} T_{m}^{q} \underset{\vec{j}}{\leftarrow} \lim _{\vec{j}}\left(X_{j, m-1}^{p, q+1}\right)^{\prime} .
$$

Here ${ }^{t} T_{m}^{q}$ is the operator obtained with the family $\left({ }^{t} T_{j, m}^{q}\right)_{j}$.

The space ${ }^{K_{0}} \varrho_{2}^{p, q}(\Omega)$ is a F.S.* space and the maps ${ }^{t} s_{j, k, m}^{p, q}$ are injective, so the sequence (3) can be also written

$$
\left(K_{0} \varrho_{2}^{p, q-1}(\Omega)\right)^{\prime} \stackrel{{ }^{\prime} T_{m+1}^{q-1}}{\leftarrow}\left(K_{0} \varrho_{2}^{p, q}(\Omega)\right)^{\prime}{ }^{\prime} T_{m}^{q} \leftarrow\left(K_{0} \varrho_{2}^{p, q+1}(\Omega)\right)^{\prime} .
$$

We are going to show that every operator ${ }^{t} T_{m}^{q}$ is equal to the dual operator ${ }^{t} T^{q}$ of $T^{q}$. We have $D\left({ }^{t} T_{m}^{q}\right) \subset D\left({ }^{t} T^{q}\right)$. If $f \in D\left({ }^{t} T_{m}^{q}\right)$, there is $k \in \mathbf{N}$ such that $f \in D\left({ }^{t} T_{k, m}^{q}\right)$ and the map $g \rightarrow\left\langle f, T_{k, m}^{q} g\right\rangle$ is continuous on the subspace $D\left(T_{k, m}^{q}\right)$ of $X_{k, m}^{p, q}$. We obtain the desired conclusion from $s_{k, m}^{p . q}\left(D\left(T^{q}\right)\right) \subset D\left(T_{k, m}^{q}\right)$ (where $s_{k, m}^{p, q}$ is the projective limit of the maps $\left.s_{k, j, m}^{p, q}, j \geqslant k\right)$.

Now we prove that $D\left({ }^{t} T^{q}\right) \subset D\left({ }^{t} T_{m}^{q}\right)$. If $f \in D\left({ }^{t} T^{q}\right)$, the map $g \rightarrow\left\langle f, T^{q} g\right\rangle$ is continuous on $D\left(T^{q}\right)$. This means that for every $m \in \mathbf{N}$, there are $k \in \mathbf{N}$ and $A>0$ such that $\left|\left\langle f, T^{q} g\right\rangle\right| \leqslant A\|g\|_{X, m, q}^{p .}$. If $g \in D\left(T_{k+1, m}^{q}\right)$, it is obvious that $s_{k, k+1, m}^{p, q}(g) \in$ $D\left(T_{k, m}^{q}\right)$ and

$$
s_{k, k+1, m+1}^{p, q+1}\left(T_{k+1, m}^{q}(g)\right)=T_{k, m}^{q}\left(s_{k, k+1, m}^{p, q}(g)\right) \in X_{k, m}^{p, q+1} .
$$

As $\Omega \subset \mathbf{D}^{2 n} \backslash\{0\}$, we can apply Proposition 2.1 .1 of $[6, \mathrm{p} .100]$ to the following situation.

The operator $T: X_{k, m}^{p, q} \rightarrow X_{k, m}^{p, q+1}$ with domain $D(T)=\left\{f \in \underset{k, m}{p, q} \mid \bar{\partial} f \in X_{k, m}^{p, q+1}\right\}$ and $T(f)=\bar{\partial} f$. The hypotheses on $a$ and $K_{k}$ show that $\mathscr{D}^{p, q}(\bar{\Omega}) \subset D(T)$ and is dense in that space for the graph norm $f \rightarrow\left(\|f\|_{X_{k, m}^{p, q}}^{2}+\|T f\|_{X_{k, m}^{p, q+1}}^{2}\right)^{1 / 2} \cdot\left(\mathscr{Q}^{p, q}(\bar{\Omega})\right.$ is the set of restrictions to $\Omega \cap \mathbf{C}^{n}$ of $(p, q)$ forms of $\mathscr{Q}^{p, q}\left(\mathbf{C}^{n}\right)$.) Then for $g \in$ $D\left(T_{k+1, m}^{q}\right)$, there is a sequence $\left(g_{l}\right)_{l \geqslant 1}$ of $\mathscr{D}^{p, q}(\bar{\Omega}) \subset D\left(T^{q}\right)$ such that

$$
\lim _{l \rightarrow+\infty}\left\|s_{k, k+1, m}^{p, q}(g)-s_{k, m}^{p, q}\left(g_{l}\right)\right\|_{X_{k, m}^{p, q}}+\left\|T\left(s_{k, k+1, m}^{p, q}(g)-s_{k, m}^{p, q}\left(g_{l}\right)\right)\right\|_{X_{k, m}^{p, q}}=0 ;
$$

that is,

$$
\lim _{l \rightarrow+\infty}\left\|s_{k, k+1, m}^{p, q}(g)-s_{k, m}^{p, q}\left(g_{l}\right)\right\|_{X_{k, m}^{p, q}}+\left\|T_{k, m}^{q}\left(s_{k, k+1, m}^{p, q}(g)\right)-T^{q}\left(g_{l}\right)\right\|_{X_{k, m}^{p, q}}=0 .
$$

Then

$$
\lim _{l \rightarrow+\infty}\left|\left\langle f, T^{q} g_{l}\right\rangle\right|=\left|\left\langle f, T_{k, m}^{q} s_{k, k+1, m}^{p, q}(g)\right\rangle\right| \leqslant A\left\|s_{k, k+1, m}^{p, q}(g)\right\|_{X_{k, m}^{p, q}} \leqslant A\|g\|_{X_{k, m+1}^{p, q}}
$$
and $f \in D\left({ }^{t} T_{m}^{q}\right)$. 
Now it is obvious that the operators ${ }^{t} T_{m}^{q}$ and ${ }^{t} T^{q}$ coincide on their common domain. We have also proved that, for $q \geqslant 2$, the sequences

$$
\left(K_{0} \varrho_{2}^{p, q+1}(\Omega)\right)^{\prime}{ }^{\prime} T^{q} \rightarrow\left(K_{0} \varrho_{2}^{p, q}(\Omega)\right)^{\prime} T^{q-1} \rightarrow\left(K_{0} \varrho_{2}^{p, q-1}(\Omega)\right)^{\prime}{ }^{\prime} T^{q-2} \rightarrow\left(K_{0} \varrho_{2}^{p, q-1}(\Omega)\right)^{\prime}
$$

are exact. The Serre-Komatsu duality lemma shows the exactitude of the sequence

$$
K_{0} \bigodot_{2}^{p, q-1}(\Omega) \stackrel{T^{q-1}}{\rightarrow} K_{0} \varrho_{2}^{p, q}(\Omega) \stackrel{T^{q}}{\rightarrow} K_{0} \varrho_{2}^{p, q+1}(\Omega) .
$$

In the case $q=1$, we must prove that $\operatorname{Im}^{t} T^{0}$ is closed to apply the Serre-Komatsu duality lemma, which shows the exactness of the sequence

$$
K_{0} \bigodot_{2}^{p, 0}(\Omega) \stackrel{T^{0}}{\rightarrow} K_{0} \bigodot_{2}^{p, 1}(\Omega) \stackrel{T^{1}}{\rightarrow} K_{0} \bigodot_{2}^{p .2}(\Omega)
$$

The method will be the following:

$\left(1^{\circ}\right)$ We use the same spaces taking $\sqrt{1+|z|^{2}}$ instead of $|z|$ in the weight functions.

$\left(2^{\circ}\right)$ We prove the exactness of the sequence

$$
0 \leftarrow\left(\operatorname{Ker} T^{0}\right)^{\prime} \leftarrow\left({ }^{K} \varrho_{2}^{p, 0}(\Omega)\right)^{\prime}{ }^{\prime} T^{0} \leftarrow\left(K_{0} \varrho_{2}^{p, 1}(\Omega)\right)^{\prime} \leftarrow \cdots .
$$

In fact, instead of $\left(\operatorname{Ker} T^{0}\right)^{\prime}$ we shall use the space $\lim _{j_{j}}\left(\operatorname{Ker} T_{j, m}^{0}\right)^{\prime}$, and it will be sufficient to prove that this is a Hausdorff space. We prove this by showing that the natural maps of this inductive limit are injective (see [9, Lemma 3, p. 372]).

As a corollary we obtain the density of the image of $\operatorname{Ker} T_{j+1, m}^{0}$ in $\operatorname{Ker} T_{j, m}^{0}$.

Let us recall that

$$
0 \rightarrow \operatorname{Ker} T_{j, m}^{0} \stackrel{J_{j}}{\rightarrow} X_{j, m}^{p, 0} \stackrel{T_{j, m}^{0}}{\rightarrow} X_{j, m-1}^{p, 1} \rightarrow \cdots
$$

is an exact sequence of Hilbert space with closed densely defined operators $T_{j, m}^{q}$. The map $J_{j}$ is the canonical injection of $\operatorname{Ker} T_{j, m}^{0}$ in $X_{j, m}^{p, 0}$.

The dual sequence is also exact and of the same type:

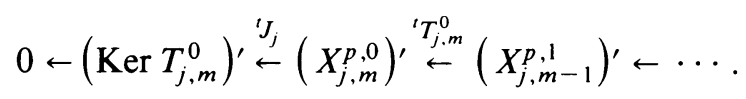

We have an inductive system of exact sequences:

$$
\begin{aligned}
& 0 \leftarrow\left(\operatorname{Ker} T_{j, m}^{0}\right)^{\prime} \quad \stackrel{{ }^{t} J_{j}}{\leftarrow} \quad\left(X_{j, m}^{p, 0}\right)^{\prime} \quad \stackrel{{ }^{\prime} T_{j, m}^{0}}{\leftarrow} \quad\left(X_{j, m-1}^{p, 1}\right)^{\prime} \leftarrow \cdots \\
& \downarrow^{\prime} r_{j} \quad \downarrow^{t} s_{j, j+1, m}^{p, 0} \quad \downarrow^{t} s_{j, j+1, m-1}^{p, 1} \\
& 0 \leftarrow\left(\operatorname{Ker} T_{j+1, m}^{0}\right)^{\prime} \stackrel{t_{j+1}}{\leftarrow} \quad\left(X_{j+1, m}^{p, 0}\right)^{\prime} \stackrel{{ }^{\prime} T_{j+1, m}^{0}}{\leftarrow} \quad\left(X_{j+1, m-1}^{p, 1}\right)^{\prime} \leftarrow \cdots
\end{aligned}
$$

with $r_{j}$ the restriction map from $\operatorname{Ker} T_{j+1, m}^{0}$ to $\operatorname{Ker} T_{j, m}^{0}$. 
A straightforward calculation shows that $\operatorname{Ker} T^{0}=\lim _{-j} \operatorname{Ker} T_{j, m}^{0}$ and that the sequence obtained by taking the inductive limit is also exact:

$$
0 \leftarrow \lim _{j}\left(\operatorname{Ker} T_{j, m}^{0}\right)^{\prime}{ }^{\prime} \leftarrow\left(K_{0} \varrho_{2}^{p .0}(\Omega)\right)^{\prime} \stackrel{T}{0}^{0}\left(K_{0} \varrho_{2}^{p .1}(\Omega)\right)^{\prime} \cdots .
$$

We only need to prove that the space $\lim _{j_{j}}\left(\operatorname{Ker} T_{j, m}^{0}\right)^{\prime}$ is a Hausdorff space. This will be done by the following lemma.

2.2.6. Lemma. The maps ${ }^{t} r_{j}$ are injective.

Proof. Let $S \in\left(\operatorname{Ker} T_{j, m}^{0}\right)^{\prime}$ such that ${ }^{t} r_{j}(S)=0$. There is $f \in\left(X_{j, m}^{p, 0}\right)^{\prime}$ such that $J_{j}(f)=S$. Then with $\tilde{f}={ }^{t} s_{j, j+1, m}^{p, 0}(f)$ we have ${ }^{t} J_{j+1}(\tilde{f})=0$.

We apply Propositions 2.3.1 and 2.3.2 of [6] with $\Omega=L_{j+1}, \varphi(z)=a(z)+$ $(j+1)^{-1} \sqrt{1+|z|^{2}}+2 m \log \left(1+|z|^{2}\right)$ and $\Psi(z)=\sigma(z)-j$. So we get $U \in$ $L_{2}^{p .1}\left(L_{j+1},-\varphi\right)$ such that:

(1) $U=0$ in $\left\{z \in L_{j+1} \mid \sigma(z)>j\right\}=L_{j+1} \backslash \bar{L}_{j}$;

(2)

$$
\tilde{f}=(-1)^{p-1} \sum_{I}^{\prime}\left(\sum_{j} \frac{\partial U_{I, j}}{\partial \bar{z}_{j}}\right) d Z_{I}
$$

(3)

$$
\int_{L_{j+1}} \sum_{I}^{\prime} \sum_{j, k} U_{I, j} \bar{U}_{I, k} \frac{\partial^{2} \varphi}{\partial z_{j} \partial \bar{z}_{k}} e^{\varphi} d \lambda \leqslant \int_{L_{j+1}}|\tilde{f}|^{2} e^{\varphi} d \lambda<+\infty .
$$

But the function $z \rightarrow c(z)=1 / 4 \sqrt{1+|z|^{2}}$ is a lower bound for the plurisubharmonicity of $\varphi$ (see [6]), so we get from $\left(3^{\circ}\right)$ that

$$
\int_{L, j} \frac{|U|^{2} e^{\varphi}}{\sqrt{1+|z|^{2}}} d \lambda=\int_{L_{j+1}} \frac{|U|^{2} e^{\varphi}}{\sqrt{1+|z|^{2}}} d \lambda<+\infty .
$$

There is a holomorphic function $\varphi_{0}$, property $(\mathrm{P})$, such that $e^{-\varphi_{0} / l} U \in\left(X_{j, m-1}^{p, 1}\right)^{\prime}$ for every $l \in \mathbf{N}^{*}$. Furthermore, we have ${ }^{t} T_{j, m}^{0}\left(e^{-\varphi_{0} / l} U\right)=e^{-\varphi_{0} / l} f$ because:

(i) We show that $e^{-\varphi_{0} / l} U \in D\left({ }^{t} T_{j, m}^{0}\right)$ : if $g \in \mathscr{Q}^{p, 1}\left(L_{j+1}\right)$ then

$$
\left|\left\langle e^{-\varphi_{0} / l} U, \bar{\partial} g\right\rangle\right|=\left|\left\langle e^{-\varphi_{0} / l} f, g\right\rangle\right| \leqslant\left\|e^{-\varphi_{0} / l} f\right\| \cdot\|g\| .
$$

This inequality remains true by taking $g \in D\left(T_{j, m}^{0}\right)$ (we use the density of $\mathscr{Q}^{p, 1}\left(L_{j+1}\right)$ in $D\left({ }^{t} T_{j, m}^{0}\right)$ for the graph norm, which obviously comes from Proposition 2.1.1 of [6]).

(ii) We prove the equality ${ }^{t} T_{j, m}^{0}\left(e^{-\varphi_{0} / l} U\right)=e^{-\varphi_{0} / l} f$ in the same way as (i).

At last we use Lebesgue's dominated convergence theorem in $0=\left\langle e^{-\varphi_{0} / l} f, h\right\rangle$ to obtain $0=\langle f, h\rangle$ for every $h \in \operatorname{Ker} T_{j, m}^{0}$. That proves $f \in\left(\operatorname{Ker} T_{j, m}^{0}\right)^{\perp}$, i.e. $S=0$.

We can now prove the following:

2.2.7. THEOREM. The sheaves $K_{0} \mathcal{H}^{p, q}$ are a Dolbeault resolution of $K_{0} \vartheta^{p, 0}$ :

$$
0 \rightarrow K_{0} \vartheta^{p, 0} \rightarrow K_{0} \mathcal{K}^{p, 0} \stackrel{\bar{\partial}}{\rightarrow} K_{0} \mathcal{H C}^{p, 1} \stackrel{\bar{\partial}}{\rightarrow} \cdots \stackrel{\bar{\partial}}{\rightarrow} K_{0} \mathcal{H C}^{p, n} \rightarrow 0 .
$$


Proof. It is sufficient to show it for germs at points in $S_{\infty}^{2 n-1}$. For such a point $U_{\infty}$ and a neighborhood $\mathcal{Q}$ of $U_{x}$, there is an open neighborhood $\Omega \subset \mathcal{Q}$ satisfying the conditions of 2.2.5.

(i) We take $\Omega=z_{0}+\Gamma \amalg(\operatorname{pr} \Gamma)_{x}$, with $\Gamma$ a convex cone containing no real line generated by an $\mathbf{R}$-base $\mathscr{H}$ of $\mathbf{R}^{2 n}=\mathbf{C}^{n}, \mathscr{H}=\left(e_{1}, \ldots, e_{2 n}\right)$, such that if $M=\left(a_{i j}\right)$ is the transfer matrix from the canonical base of $\mathbf{C}^{n}$ to the new one, the new coordinates are $\left(x_{l}^{\prime}\right)$, where

$$
x_{l}^{\prime}=\sum_{k=1}^{2 n} a_{l, k} x_{k} \quad \text { with } z=\left(z_{1}, \ldots, z_{n}\right), z_{p}=x_{2 p-1}+i x_{2 p} .
$$

(ii) The function $\sigma(z)=\sum_{p=1}^{2 n}\left(x_{p}^{\prime}-x_{0, p}^{\prime}\right)^{-1}$ (where the coordinates of $z_{0}$ are $\left(x_{0, p}^{\prime}\right)_{1 \leqslant p \leqslant 2 n}$ in the new base) is strictly plurisubharmonic in $z_{0}+\Gamma$, belongs to $e^{\infty}\left(z_{0}+\Gamma\right)$, and satisfies the conditions of 2.2.5.

(iii) We can find a base $\beta$ such that $\Omega$ has property (P). Now Theorem 2.2.7 is a direct corollary of Theorem 2.2.5.

2.3. Mean periodic functions of $\mathcal{H}_{C}(\Omega)$. We will denote by $\Gamma$ a convex sector of $\mathbf{C}$ containing no real line and take $K_{0}=\{0\}$. Then $a \equiv 0$. We consider the closed convex (noncompact) set $\Omega=\{z \in \mathbf{C} \mid-\operatorname{Im} z \zeta \leqslant 0 \forall \zeta \in \Gamma\}=\Omega(\Gamma, 0)$.

Let us remark that if $\zeta$ is a given point of a conic neighborhood of $\Omega$ and $f$ is a given function of $\mathcal{H}_{c}(\Omega)$, the function $\tau_{\zeta}(f): z \rightarrow f(z+\zeta)$ belongs to $\mathcal{H}_{c}(\Omega)$. So we can define, for $\mu \in \mathcal{H}_{c}^{\prime}(\Omega)$, the function $\mu * f: \zeta \rightarrow(\mu * f)(\zeta)=\left\langle\mu, \tau_{\zeta}(f)\right\rangle$, and it is easy to prove that $\mu * f \in \mathcal{H}_{c}(\Omega)$.

If $\mu$ and $\nu \in \mathcal{H}_{c}^{\prime}(\Omega)$, as usual, we have $\langle\mu, \nu * f\rangle=\langle\nu, \mu * f\rangle$, so we can define $\mu * \nu$ by $\langle\mu * \nu, f\rangle=\langle\mu, \nu * f\rangle$, and we have $\mathscr{F}(\mu * \nu)=\mathscr{F}(\mu) \mathscr{F}(\nu)$. Now $\mathcal{H}_{c}^{\prime}(\Omega)$ is a convolution algebra isomorphic to the algebra $\operatorname{Exp}_{c}(\Gamma, 0)\left(\operatorname{Exp}_{c}(\Gamma, 0)\right.$ with the usual product of functions).

As in Chapter I, we can define invariant subspaces of $\mathcal{H}_{C}(\Omega)$ and mean periodic function. The problem of spectral analysis is solved as in Chapter I.

Our duality method (for the spectral synthesis) needs the introduction of the following spaces of $e^{\infty}$ functions.

2.3.1. Definition. Let $\left(\Gamma_{k}\right)_{k \geqslant 1}$ be an exhaustion of $\Gamma$. We note $\operatorname{Exp}_{c}^{\infty}(\Gamma)$ the space of $C^{\infty}$ functions in $\Gamma$ such that for every $k$ and $j \in \mathbf{N}$,

$$
q_{k, j}(f)=\sup _{z \in \Gamma(k)}\left|\frac{\partial^{j} f}{\partial z^{j}}(z) e^{-|z| / k}\right|<+\infty
$$

(with $\Gamma(k)=\Gamma_{k} \cap\{z \in \mathbf{C}|| z \mid>1 / k\}$ ).

This space is independent from the chosen exhaustion and has a unique topology of Fréchet space defined by the family of seminorms $q_{k, j}$ and by the seminorm of uniform convergence of $\partial^{p+q} f / \partial z^{p} \partial \bar{z}^{q}(p \geqslant 0, q \geqslant 0)$ on every compact set of $\Gamma$.

\subsubsection{Proposition. The Schwartz space $\mathscr{D}(\Gamma)$ is dense in $\operatorname{Exp}_{c}^{\infty}(\Gamma)$.}

Proof. This is a standard proof.

Therefore the dual space of $\operatorname{Exp}_{c}^{\infty}(\Gamma)$ is a space of distributions. 
2.3.3. RemarK. We have $\operatorname{Exp}_{c}(\Gamma, 0) \subset \operatorname{Exp}_{c}^{\infty}(\Gamma)$. In fact, $\operatorname{Exp}_{c}(\Gamma, 0)$ is the subset of holomorphic functions of $\operatorname{Exp}_{c}^{\infty}(\Gamma, 0)$. If $f \in \operatorname{Exp}_{c}(\Gamma, 0), z \in \Gamma(k)$ and $0<r<$ $d\left(\Gamma(k), \Gamma(k+1)^{c}\right)$, there is $B_{k}(f, r)>0$ such that

$$
\sum_{p \geq 0} \frac{r^{p}}{p !}\left|\frac{\partial^{p} f}{\partial z^{p}}(z)\right| \leqslant B_{k}(f, r) e^{|z| / k}
$$

2.3.4. Lemma. Let $\omega$ be an open set of $\mathbf{C}$ and $K$ a compact subset of $\omega$. There is $C>0$ such that for every $u \in \mathcal{E}^{1}(\omega)$ we have

$$
\operatorname{Sup}_{K}|u| \leqslant C\left(\operatorname{Sup}_{\omega}\left|\frac{\partial u}{\partial \bar{z}}\right|+\int_{\omega}|u| d \lambda\right) .
$$

Proof. Classical: Let $\varphi$ be a function of $O(\omega)$ equal to 1 on a neighborhood of $K$ with value in $[0,1]$. For $a \in K$ we have

$$
u(a)=\frac{1}{2 i \pi} \int_{\omega} \frac{\partial \varphi}{\partial \bar{z}} \frac{u}{z-a} d z \wedge d \bar{z}+\frac{1}{2 i \pi} \int_{\omega} \varphi \frac{\partial u}{\partial \bar{z}} \frac{1}{z-a} d z \wedge d \bar{z}
$$

which gives the inequality.

2.3.5. THEOREM. The following sequence is exact:

$$
0 \rightarrow \operatorname{Exp}_{c}(\Gamma, 0) \rightarrow \operatorname{Exp}_{c}^{\infty}(\Gamma) \stackrel{\partial / \partial \bar{z}}{\rightarrow} \operatorname{Exp}_{c}^{\infty}(\Gamma) \rightarrow 0 .
$$

Proof. We need only to prove the surjectivity of $\partial / \partial \bar{z}$. We have $\operatorname{Exp}_{c}^{\infty}(\Gamma) \subset$ ${ }^{0} \mathcal{L}_{2}\left(\Gamma \amalg(\operatorname{pr} \Gamma)_{\infty}\right)$, so by 2.2 .5 if $f \in \operatorname{Exp}_{c}^{\infty}(\Gamma)$ there is $v \in{ }^{0} \mathcal{L}_{2}\left(\Gamma \amalg(\operatorname{pr} \Gamma)_{\infty}\right)$ such that $\partial v / \partial \bar{z}=f$ (in the sense of distribution theory) but $v$ is a $C^{\infty}$ function because $f$ is also $e^{\infty}$. The function $v$ is such that for every $k \geqslant 1$,

$$
\int_{\Gamma(k)}|v(z)|^{2} \frac{e^{-|z| / k}}{\left(1+|z|^{2}\right)^{2}} d \lambda(z)<+\infty .
$$

Let us fix $k$ and $0<r<d\left(\Gamma(k), \Gamma(k+1)^{c}\right)$. For $z_{0} \in \Gamma(k)$, we set $u(\zeta)=$ $v\left(z_{0}+\zeta\right)$. This is a $C^{\infty}$ function in the disk $D(0, r)$. Then by 2.3 .4 we have

$$
\left|v\left(z_{0}\right)\right| \leqslant C\left(\sup _{\left|z-z_{0}\right| \leqslant r}\left|\frac{\partial v}{\partial \bar{z}}(z)\right|+\int_{\left|z-z_{0}\right| \leqslant r}|v(z)| d \lambda(z)\right) .
$$

Then the Cauchy-Schwarz inequality shows that $q_{k, 0}(v)<+\infty$. The Pompeiu formula gives

$$
\frac{\partial v}{\partial z}\left(z_{0}\right)=\frac{1}{2 i \pi}\left\{\int_{\left|z-z_{0}\right|=r} \frac{1}{z-z_{0}} \frac{\partial v}{\partial z} d z+\int_{\left|z-z_{0}\right| \leqslant r} \frac{1}{z-z_{0}} \frac{\partial^{2} v}{\partial z \partial \bar{z}} d z \wedge d \bar{z}\right\},
$$

but

$$
d\left(\frac{v}{z-z_{0}}\right)=\left\{\frac{1}{z-z_{0}} \frac{\partial v}{\partial z}-\frac{v}{\left(z-z_{0}\right)^{2}}\right\} d z+\frac{1}{z-z_{0}} \frac{\partial v}{\partial \bar{z}} d \bar{z}
$$


SO

$$
\begin{aligned}
\frac{\partial v}{\partial z}\left(z_{0}\right)=\frac{1}{2 i \pi}\left\{\int_{\left|z-z_{0}\right|=r} \frac{v(z)}{\left(z-z_{0}\right)^{2}} d z-\int_{\left|z-z_{0}\right|=r} \frac{1}{z-z_{0}} \frac{\partial v}{\partial \bar{z}} d \bar{z}\right. \\
\left.\quad+\int_{\left|z-z_{0}\right| \leqslant r} \frac{1}{z-z_{0}} \frac{\partial^{2} v}{\partial z \partial \bar{z}} d z \wedge d \bar{z}\right\} .
\end{aligned}
$$

As $f \in \operatorname{Exp}_{c}^{\infty}(\Gamma)$, and we have proved that $q_{k, 0}(v)<+\infty$, we have $q_{k, 1}(v)<+\infty$.

The inequalities $q_{k, j}(v)<+\infty$ for every $j$ are obtained by an easy induction procedure.

Let us recall here the notion of multiplicity variety of an ideal (see [2 and 3]).

Let $\rho=\left(\rho_{1}, \ldots, \rho_{n}\right)$ be an $n$-tuple of functions of $\operatorname{Exp}_{c}(\Gamma, 0)$. We call $I=$ $\left(\rho_{1}, \ldots, \rho_{n}\right)$ the multiplicity variety of the ideal and we denote by $V(I)$ the set of pairs $(z, m) \in \Gamma \times \mathbf{N}$ such that $\rho_{j}(z)=0$ for every $j$, where $m$ is multiplicity of the zero $z$ of $\rho$.

For $\varepsilon>0$, we let

$$
S(k, \rho, \varepsilon)=\left\{\left.z \in \Gamma(k)\left|\sum_{j=1}^{n}\right| \rho_{j}(z)\right|^{2}<\varepsilon^{2}\right\} .
$$

If $\left(\Gamma_{k}\right)_{k \geqslant 1}$ is an exhaustion of $\Gamma$, we shall let $\Gamma(0)=\varnothing$ and

$$
S_{k}=\Gamma(k) \backslash \Gamma(k-1) k \geqslant 1 \quad(\text { for } k \geqslant 1) .
$$

If $\rho=\left(\rho_{1}, \ldots, \rho_{n}\right)$ is an $n$-tuple of functions, $V_{1}(\rho)$ denotes the set of common zeroes of the functions $\rho_{j}(1 \leqslant j \leqslant n)$.

We shall introduce some useful technical conditions to prove our result 2.3.11.

2.3.6. Conditions. Let $\rho=\left(\rho_{1}, \ldots, \rho_{n}\right)$ be an $n$-tuple of functions of $\operatorname{Exp}_{c}(\Gamma, 0)$. We suppose that there is an exhaustion $\left(\Gamma_{k}\right)_{k \geqslant 1}$ of $\Gamma$ such that:

(1) For every $k \geqslant 1$,

$$
r_{1, k}=\inf _{z \in V_{1}(\rho) \cap S_{k}} d\left(z, S_{k}^{c}\right)>0
$$

(2) For every $k \geqslant 1$,

$$
r_{2, k}=\inf \left\{d\left(z, z^{\prime}\right) \mid z \text { and } z^{\prime} \in V_{1}(\rho) \cap S_{k} ; z \neq z^{\prime}\right\}>0 \text {. }
$$

(3) There are two sequences of strictly positive numbers $\left(r_{k}\right)_{k \geqslant 1}$ and $\left(\varepsilon_{m}\right)_{m \geqslant 1}$ such that:

(a) $0<r_{k}<\inf \left(r_{1, k}, r_{2, k}\right)$ for every $k \geqslant 1$;

(b) $S\left(m, \rho, \varepsilon_{m}\right)$ is a subset of $\cup_{1 \leqslant k \leqslant m} \cup_{z \in V_{1}(\rho) \cap s_{k}} D\left(z, r_{k} / 2\right)$ for every $m \geqslant 1$.

(4) $M_{k}=\operatorname{Sup}\left\{m_{z} \mid\left(z, m_{z}\right) \in V(\rho)\right.$ and $\left.z \in \Gamma(k)\right\}<+\infty$ for every $k$.

Let us recall that if $\rho=\left(\rho_{1}, \ldots, \rho_{n}\right)$ is a given $n$-tuple of functions of $\operatorname{Exp}_{c}(\Gamma, 0)$, we call $I_{\text {loc }}\left(\rho_{1}, \ldots, \rho_{n}\right)$ the local ideal generated by $\left(\rho_{1}, \ldots, \rho_{n}\right)$ this is the closed ideal of functions of $\operatorname{Exp}_{c}(\Gamma, 0)$ which belong to the section of the sheaf of ideals generated by $\rho_{1}, \ldots, \rho_{n}$. This ideal contains $I\left(\rho_{1}, \ldots, \rho_{n}\right)$. Here is an example of ideals such that $I=I_{\text {loc }}($ see [2]).

2.3.7. Definition. $A$ function $\rho \in \operatorname{Exp}_{c}(\Gamma, 0)$ is called slowly decreasing if there is an exhaustion $\left(\Gamma_{k}\right)_{k \geqslant 1}$ of $\Gamma$ and a sequence $\left(\varepsilon_{k}\right)_{k \geqslant 1}$ of strictly positive numbers such that the set $S\left(k, \rho, \varepsilon_{k}\right)$ has its connected components relatively compact in $\Gamma(k)$ and with bounded diameter. 
EXAmPle. $\Gamma=\{z \in \mathbf{C}$ such that $|\operatorname{Arg} z|<\pi / 4\}$ and $\rho(z)=\prod_{n \geqslant 1}\left(1-z / 2^{n}\right)$.

This function satisfies Conditions 2.3.6.

2.3.8. Proposition. If $\rho \in \operatorname{Exp}_{c}(\Gamma, 0)$ is slowly decreasing, we have $I(\rho)=I_{\text {loc }}(\rho)$.

Proof. See [2].

2.3.9. Definition. Let $I=I(\rho)$ (with $\left.\rho=\left(\rho_{1}, \ldots, \rho_{n}\right)\right)$ be a finitely generated ideal of $\operatorname{Exp}_{c}(\Gamma, 0)$ and $V(I)=\left\{\left(z_{p}, m_{p}\right)_{p \geqslant 0}\right\}$ its multiplicity variety. We denote by $I_{\infty}(\rho)$ the following ideal of $\operatorname{Exp}_{c}^{\infty}(\Gamma)$ :

$$
I_{\infty}(\rho)=\left\{f \in \operatorname{Exp}_{c}^{\infty}(\Gamma) \mid \frac{\partial^{j+q} f}{\partial z^{j} \partial \bar{z}^{q}}\left(z_{p}\right)=0,0 \leqslant j \leqslant m_{p-1}(p, q) \in \mathbf{N}^{2}\right\} .
$$

It is obvious that $I_{\infty}(\rho)$ is a closed ideal of $\operatorname{Exp}_{c}^{\infty}(\Gamma)$. When $n=1$, this ideal is equal to the set of functions $f \in \operatorname{Exp}_{c}^{\infty}(\Gamma)$ such that $f$ can be written $f=\rho u$ with a $\bigodot^{\infty}$ function $u$ (this follows easily from Taylor's formula).

Let us now recall the interpolation theorem.

THEOREM. Let $\rho=\left(\rho_{1}, \ldots, \rho_{n}\right)$ be an $n$-tuple of functions of $\operatorname{Exp}_{c}(\Gamma, 0)$ verifying Conditions 2.3.6. Let $V(I)=\left\{\left(z_{p}, m_{p}\right)_{p \geqslant 0}\right\}$ be the multiplicity variety of the ideal $I=I\left(\rho, \ldots, \rho_{n}\right)$. Let $\left\{a_{p, j}: p \geqslant 0,0 \leqslant j \leqslant m_{p}-1\right\}$ be a sequence of complex numbers such that, for every $k \geqslant 1$,

$$
\operatorname{Sup}_{\substack{z_{p} \in \Gamma(k) \\ 0 \leqslant j \leqslant m_{p}-1}}\left|\frac{a_{p, j}}{j !} e^{-\left|z_{p}\right| / k}\right|<+\infty .
$$

Then there is a function $h \in \operatorname{Exp}_{c}(\Gamma, 0)$ such that $\partial^{j} h\left(z_{p}\right) / \partial z^{j}=a_{p, j}($ for every $p \in \mathbf{N}$ and $0 \leqslant j \leqslant m_{p}-1$ ).

We emphasize that this theorem can be proved by direct classical means.

We consider the sequences

$$
\begin{gathered}
0 \rightarrow \operatorname{Exp}_{c}(\Gamma, 0) \rightarrow \operatorname{Exp}_{c}^{\infty}(\Gamma) \stackrel{\partial / \partial \bar{z}}{\rightarrow} \operatorname{Exp}_{c}^{\infty}(\Gamma) \rightarrow 0, \\
0 \rightarrow I_{10 c}\left(\rho_{1}, \ldots, \rho_{n}\right) \stackrel{J}{\rightarrow} I_{\infty}\left(\rho_{1}, \ldots, \rho_{n}\right) \stackrel{\partial / \partial \bar{z}}{\rightarrow} I_{\infty}\left(\rho_{1}, \ldots, \rho_{n}\right) \rightarrow 0,
\end{gathered}
$$

and

$$
0 \rightarrow \frac{\operatorname{Exp}_{c}(\Gamma, 0)}{I_{\text {loc }}\left(\rho_{1}, \ldots, \rho_{n}\right)} \stackrel{J}{\rightarrow} \frac{\operatorname{Exp}_{c}^{\infty}(\Gamma)}{I_{\infty}\left(\rho_{1}, \ldots, \rho_{n}\right)} \stackrel{\bar{\partial}}{\rightarrow} \frac{\operatorname{Exp}_{c}^{\infty}(\Gamma)}{I_{\infty}\left(\rho_{1}, \ldots, \rho_{n}\right)} \rightarrow 0
$$

which is the quotient of (1) by (2).

We have the following duality type result.

2.3.10. TheOREM. With the above notations and Conditions 2.3.6 the interpolation theorem is equivalent to the exactness of sequence (3).

Proof. (a) The interpolation theorem implies the exactness of (3). Let us show first that the interpolation theorem implies the exactness of (2). Let $f \in I_{\infty}(\rho)$ such that $\partial f / \partial \bar{z}=0$. We have $f \in \operatorname{Exp}_{c}(\Gamma, 0)$ and it is obvious that $f \in I_{\text {loc }}\left(\rho_{1}, \ldots, \rho_{n}\right)$. Let $g \in I_{\infty}(\rho)$. By the exactness of (1) there is $u \in \operatorname{Exp}_{c}^{\infty}(\Gamma)$ such that $\partial u / \partial \bar{z}=g$. 
By the interpolation theorem there is $h \in \operatorname{Exp}_{c}(\Gamma, 0)$ such that $\partial^{j} h\left(z_{p}\right) / \partial z^{j}=$ $-\partial^{j} u\left(z_{p}\right) / \partial z^{j}$ for every $p$ and $j, 0 \leqslant j \leqslant m_{p}-1$. So $u+h \in I_{\infty}(\rho)$ and $\partial(u+h) / \partial \bar{z}$ $=g$. The exactness of (3) follows easily.

(b) The exactness of (3) implies the interpolation theorem. For every $k$, let $\varphi_{k}$ be a $C^{\infty}$ function with values in $[0,1]$, support in $D\left(0, r_{k}\right)$, and equal to 1 in a neighborhood of $D\left(0, r_{k} / 2\right)$. The function

$$
\Psi(z)=\sum_{k=1}^{+\infty} \sum_{p \in S_{k}} \sum_{j=0}^{m_{p}-1} \frac{a_{p, j}}{j !}\left(z-z_{p}\right)^{j} \varphi_{k}\left(z-z_{p}\right)
$$

belongs to $\operatorname{Exp}_{c}^{\infty}(\Gamma)$. Let $\stackrel{\circ}{\Psi}$ be its equivalence class in $\operatorname{Exp}_{c}^{\infty}(\Gamma) / I_{\infty}\left(\rho_{1}, \ldots, \rho_{n}\right)$. We have $\bar{\partial}(\stackrel{\oplus}{\Psi})=0$. So there is $h^{\circ} \in \operatorname{Exp}_{c}^{\infty}(\Gamma) / I_{\text {loc }}\left(\rho_{1}, \ldots, \rho_{n}\right)$ such that $J(\stackrel{\circ}{h})=\stackrel{\circ}{\Psi}$ and any $h \in \grave{h}$ is convenient for the interpolation.

2.3.11. THEOREM Let $\rho=\left(\rho_{1}, \ldots, \rho_{n}\right)$ be an $n$-tuple of functions of $\operatorname{Exp}_{c}(\Gamma, 0)$ satisfying Conditions 2.3.6. Then (3) is exact.

Proof. We have to show the exactness of (2). The exactness of (3) is then an immediate consequence of it.

We denote by $T$ the densely defined operator $\partial / \partial \bar{z}$ of (1) and by $T_{2}$ its restriction to $I_{\infty}(\rho)$ with domain $D\left(T_{2}\right)=D(T) \cap I_{\infty}(\rho)$. To show the surjectivity of $T_{2}$, we have to prove that its dual operator ${ }^{t} T_{2}$ is injective and has weakly closed range (see $[10$, pp. 84, 100]).

Let us recall that $D\left({ }^{t} T_{2}\right)=\left\{y^{\prime} \in I_{\infty}^{\prime}(\rho)\right.$ such that the map $D\left(T_{2}\right) \rightarrow \mathbf{C}, x \rightarrow$ $\left\langle y^{\prime}, T_{2} x\right\rangle$ is continuous $\}$.

(a) Injectivity of ${ }^{t} T_{2}$. Let $f \in I_{\infty}(\rho) \cap \mathcal{O}(\Gamma)$. It is obvious that there are $n$ functions $h_{1}, \ldots, h_{n}$ of $\mathscr{D}(\Gamma)$ such that $f=\sum_{j=1}^{n} \rho_{j} h_{j}$. The exactness of (1) shows that there is $g_{j} \in \operatorname{Exp}_{c}^{\infty}(\Gamma)$ such that $\partial g_{j} / \partial \bar{z}=h_{j}$. Then $g=\Sigma_{j} \rho_{j} g_{j}$ satisfies $T_{2}(g)=f$. This shows the injectivity of ${ }^{t} T_{2}$.

(b) Let $E_{1}$ be the following subspace of $\operatorname{Exp}_{c}^{\infty}(\Gamma)$ :

$$
E_{1}=\left\{f \in \operatorname{Exp}_{c}^{\infty}(\Gamma) \mid \forall q \geqslant 1, \forall p \geqslant 0,0 \leqslant j \leqslant m_{p}-1, \frac{\partial^{q+j} f}{\partial z^{j} \partial \bar{z}^{q}}\left(z_{p}\right)=0\right\} .
$$

Then $E_{1}$ is a closed subspace of $\operatorname{Exp}_{c}^{\infty}(\Gamma)$, and $I_{\infty}(\rho)=E_{2}$ has a topological supplementary in $E_{1}$ because the following map is a continuous projection of $E_{1}$ onto $E_{2}$ :

$$
\begin{gathered}
L: E_{1} \rightarrow E_{2}, \\
f \rightarrow z \rightarrow L(f)(z)=f(z)-\sum_{k=1}^{+\infty} \sum_{p \in S_{k}} \sum_{j=0}^{m_{p}-1} \frac{a_{p, j}}{j !}\left(z-z_{p}\right)^{j} \varphi_{k}\left(z-z_{p}\right)
\end{gathered}
$$

(with $a_{p, j}=\partial^{j} f\left(z_{p}\right) / \partial z^{j}$ and $\varphi_{k}$ defined in part (b) of the proof of 2.3.10).

The exactness of (1) shows that the densely defined operator $T_{1}=\partial / \partial \bar{z}$ is surjective from $E_{1}$ to $E_{2}$. It is obvious that $D\left({ }^{t} T_{2}\right)=D\left({ }^{t} T_{1}\right)$.

Furthermore, the weak dual $E_{2}^{\prime}$ of $E_{2}$ is isomorphic to $E_{1}^{\prime} / E_{2}^{\perp}$ when $E_{1}^{\prime} / E_{2}^{\perp}$ has the quotient topology of the weak topology of $E_{1}^{\prime}$ (see [19]). 
Let $\pi^{*}$ be the canonical projection of $E_{1}^{\prime}$ on $E_{1}^{\prime} / E_{2}^{\perp}$. It is obvious that $\pi^{*}\left(\operatorname{Im}^{t} T_{1}\right)$ $=\operatorname{Im}^{t} T_{2}$. Let us show that $E_{2}^{\perp} \subset \operatorname{Im}^{t} T_{1}$. Let $\mu \in E_{2}^{\perp}$. We have

$$
\mu=\sum_{p} \sum_{k+m \leqslant \alpha_{p}} a_{k, m} \frac{\partial^{k+m}}{\partial z^{k} \partial \bar{z}^{m}} \delta_{z_{p}}
$$

and it suffices to show that for every $p$, the Dirac measure $\delta_{z_{p}}$ belongs to $\operatorname{Im}^{t} T_{1}$.

Using a rotation we can suppose that $\Gamma=\{z \in \mathbf{C}|| \operatorname{Arg} z \mid<\gamma<\pi / 2\}$. The distribution $G=\pi^{-1} \exp \left\{-\left(z-z_{p}\right)^{2}\right\} \times\left(z-z_{p}\right)^{-1} d \lambda$ belongs to $\left(\operatorname{Exp}_{c}^{\infty}(\Gamma)\right)^{\prime}$. Furthermore, $G \in \operatorname{Dom}^{\prime} T_{1}$ and ${ }^{t} T_{1} G=\delta_{z_{p}}$. The surjectivity of $T_{1}$ and the inclusion $E_{2}^{\perp} \subset \operatorname{Im}^{\prime} T_{1}$ show that ${ }^{t} T_{2}$ has a weakly closed range.

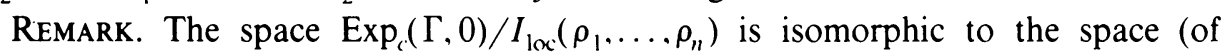
sequences)

$$
S=\left\{\left(a_{p, j}\right)_{p \in \mathbf{N}: 0 \leqslant j \leqslant m_{p},}\left|\sup _{z_{p} \in l_{1}(\rho) \cap \Gamma(k)}\right| \frac{a_{p \cdot j}}{j !} e^{-\left|z_{p}\right| / k} \mid<+\infty\right\} .
$$

The space $\operatorname{Exp}_{c}^{\infty}(\Gamma) / I_{\infty}\left(\rho_{1}, \ldots, \rho_{n}\right)$ is isomorphic to a closed subspace of the space

$$
S_{1}=\left\{\left(a_{p, q, j}\right)_{p, q ; 0 \leqslant j \leqslant m_{p}-1}\left|\forall q, \sup _{z_{n} \in l_{1}(\rho) \cap \Gamma_{h}}\right| \frac{a_{p \cdot q, j}}{j !} e^{-\left|z_{p}\right| / k} \mid<+\infty\right\} .
$$

So the $\bar{\partial}$ operator can be viewed as

$$
\left(a_{p, q . j}\right)_{p . q: 0 \leqslant j \leqslant m_{p},-1} \stackrel{\bar{a}}{\rightarrow}\left(q a_{p, q . j}\right)_{p . q: 0 \leqslant j \leqslant m_{p}-1}
$$

and its kernel is the space of sequences to be interpolated.

2.3.12. COROLlaRY. With conditions 2.3 .6 the interpolation theorem is true.

We are going to apply these results to mean periodic functions of $\mathfrak{H}_{c}(\Omega)$.

2.3.13. THEOREM. Let $\left(\mu_{j}\right)_{1 \leqslant j \leqslant n}$ be $n$ analytic functionals of $\mathcal{H}_{c}^{\prime}(\Omega)$ such that $\left(\widetilde{\mathscr{T}}\left(\mu_{j}\right)\right)_{1 \leqslant j \leqslant n}$ satisfies Conditions 2.3.6 and such that

$$
I_{\text {loc }}\left(\widetilde{Y}\left(\mu_{1}\right), \ldots, \widetilde{F}\left(\mu_{n}\right)\right)=I\left(\widetilde{F}\left(\mu_{1}\right), \ldots, \mathscr{F}\left(\mu_{n}\right)=I\right. \text {. }
$$

Then every function $g \in \mathcal{H}_{c}(\Omega)$ such that $\mu_{1} * g=\cdots=\mu_{n} * g=0$ can be written

$$
g(z)=\sum_{p}\left(\sum_{k \leqslant m_{p}} b_{k, p} z^{p}\right) e^{i z \zeta_{p}} \quad\left(\text { with }\left(\zeta_{p}, m_{p}\right) \in V(I)\right)
$$

with convergent power series in $\mathcal{H}_{c}(\Omega)$.

Proof. We set $\rho_{k}=\mathscr{F}\left(\mu_{k}\right)$. The exactness of (3) and the Serre-Komatsu lemma [9] show the exactness of the following sequence:

$$
0 \leftarrow\left[\frac{\operatorname{Exp}_{c}(\Gamma, 0)}{I\left(\rho_{1}, \ldots, \rho_{n}\right)}\right]^{\prime} \leftarrow\left[\frac{\operatorname{Exp}_{c}^{\infty}(\Gamma)}{I_{\infty}\left(\rho_{1}, \ldots, \rho_{n}\right)}\right]^{\prime} \stackrel{t_{\bar{g}}}{\leftarrow}\left[\frac{\operatorname{Exp}_{c}^{\infty}(\Gamma)}{I_{\infty}\left(\rho_{1}, \ldots, \rho_{n}\right)}\right]^{\prime},
$$


that is

$$
0 \leftarrow\left(I\left(\rho_{1}, \ldots, \rho_{n}\right)\right)^{\perp} \leftarrow\left(I_{\infty}\left(\rho_{1}, \ldots, \rho_{n}\right)\right)^{\perp} \stackrel{t_{\bar{g}}}{\leftarrow}\left(I_{\infty}\left(\rho_{1}, \ldots, \rho_{n}\right)\right)^{\perp},
$$

so

$$
\left(I\left(\rho_{1}, \ldots, \rho_{n}\right)\right)^{\perp}=\frac{\left(I_{\infty}\left(\rho_{1}, \ldots, \rho_{n}\right)\right)^{\perp}}{t_{\bar{\partial}\left(\left(I_{\infty}\left(\rho_{1}, \ldots, \rho_{n}\right)\right)^{\perp}\right)}},
$$

and it suffices to remember that $\left(I_{\infty}\left(\rho_{1}, \ldots, \rho_{n}\right)\right)^{\perp}$ is (isomorphic to) a space of distributions.

\section{BIBLIOGRAPHY}

1. E. Amar, $\bar{\partial}$ cohomologie $C^{\infty}$ et applications, Prep. Univ. Paris Sud 80 T. 1.

2. C. A. Bernstein and B. A. Taylor, $A$ new look at interpolation theory for entire functions of one variable, Adv. in Math. 33 (1979), 109-143.

3. L. Ehrenpreis, Mean periodic functions. I, Amer. J. Math. 77 (1955), 293-298; Addendum, ibid. 77 (1955), 731-733.

4. R. Gay, Division des fonctionelles analytiques et fonctions entières de type exponentiel de plusieurs variables, Th. Sc. Math., Strasbourg, 1976.

5. K. Hoffman, Banach spaces of analvtic functions, Prentice-Hall, Englewood Cliffs, N.J., 1962.

6. L. Hörmander, $L^{2}$-estimates and existence theorems for the $\bar{\partial}$ operator, Acta Math. 113 (1965), $89-152$

7. An introduction to complex analysis in several variables, 2nd ed., North-Holland, Amsterdam, 1973.

8. T. Kawaï, On the theory of Fourier hyperfunctions and its application to partial differential equations with constant coefficient, J. Fac. Sci. Univ. Tokyo Set. IA Math. 17 (1970), 467-517.

9. H. Komatsu, Projective and injective limit of weakly compact sequences of locally convex spaces, $\mathrm{J}$. Math. Soc. Japan 19 (1967), 366-383.

10. G. Kothe, Topological vector spaces. II, Grundlehren Math. Wiss, Band 237, Springer-Verlag, New York, 1979.

11. P. Lelong, Fonctionnelles analytiques et fonctions entières ( $n$ variables), Univ. Montréal Press, 1968.

12. B. Malgrange, Existence et approximation des solutions des équations aux dérivées partielles et des équations de convolution, Ann. Inst. Fourier (Grenoble) 6 (1955-1956), 271-335.

13. M. Morimoto, Analytic functionals with non compact carriers. Tokyo J. Math. 1 (1978), 77-103.

14. J. W. de Roever, Fourier transform of holomorphic functions and application to Newton interpolation series. I, Publ. Math. Centrum, Amsterdam, 1974.

15. Fourier transform of holomorphic functions and application to Newton interpolation series. II,

T. W 148, Math. Centrum, Amsterdam, 1975.

16. Complex Fourier transformation and analytic functionals with unbounded carriers, Math. Centre Tracts, 89, Math. Centrum, Amsterdam, 1978.

17. Y. Sabury, Vanishing theorems of cohomology groups with coefficients in sheaves of holomorphic functions with bound, Proc. Japan Acad. Ser. A Math. Sci. 54-59 (1978), 274-278.

18. L. Schwartz, Théorie générale des fonctions moyenne-periodiques, Ann. of Math. (2) 48 (1947), $857-929$.

19. F. Treves, Topological vector spaces, distributions and kernels, Academic Press, New York, 1967.

20. V. V. Zarinov, Laplace transformation of Fourier hyperfunctions and related classes of analytic functionals. I, Theoret. and Math. Phys. 33 (1978), 1027-1039.

21. Laplace transformation of Fourier hyperfunctions and related classes of analytic functionals. II, Theoret. and Math. Phys. 37 (1979), 843-855.

Universite DE Bordeaux I, U.E.R. DE Mathematiques et D'Informatique, 33405 Talence, FRANCE 